\title{
Buckling and Vibration of Carbon Nanotubes Embedded in Polyethylene Polymers
}

\author{
By \\ Dai Shi

\begin{abstract}
A thesis submitted to the Faculty of Graduate Studies

in partial fulfilment of the requirements for the degree of

Master of Science
\end{abstract}

Department of Mechanical and Manufacturing Engineering Faculty of Engineering University of Manitoba

Winnipeg, Manitoba

July 2011

(C) Copyright

2011, Dai Shi 


\begin{abstract}
The potential of filling carbon nanotubes in polymers has been widely acknowledged for composites with exceptional new properties owing to the high strength of carbon nanotubes. In the thesis, by employing Materials Studio 4.0 software, the buckling behaviour and vibration of polyethylene and carbon nanotube matrix composites are first discussed using molecular mechanics simulations. The research is aimed to acquire a high strength design of PE-CNT matrix with proper PE/CNT ratio as well as discovering the dynamic characteristics of the PE-CNT composites. Investigation results show that as the number of PE chains increases, the buckling strain and the resonance frequency will decrease. Van der Waals forces are collected to explain the relation of the PE chains to the buckling strain and the resonance frequency of the composites.
\end{abstract}




\section{Acknowledgments}

I would like to express my deepest gratitude to Professor Quan Wang. It was my privilege to be his graduate student. I greatly appreciate his guidance on this research, his academic moral character, and his efforts on research progress.

I also want to thank Professor Peng, Dr. Luo, and Dr. Xing. Professor Peng and Dr. Luo have taught me both in undergraduate and graduate studies. Their guidance in simulation method and vibrations are extremely helpful in my graduate research. Dr. Xing brings me to an attractive and amazing world of biomaterials.

I would like to thank Mr. Nan Wu and Mr. Zhaoyang Liang. I obtained a lot of necessary knowledge on mechanics, calculation and simulation software from them.

Thanks must be given to my parents - my mother Mrs. Yaguang (Catherine) Dong and my father Mr. Jianji (James) Shi. They gave me the unconditional love, support, encouragement and help. 


\section{Contents}

\section{Front Matter}

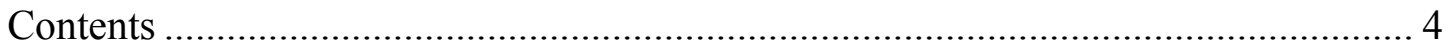

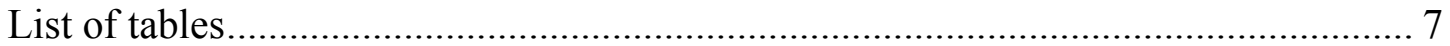

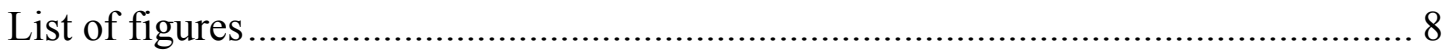

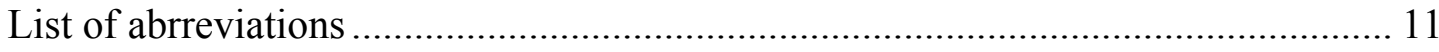

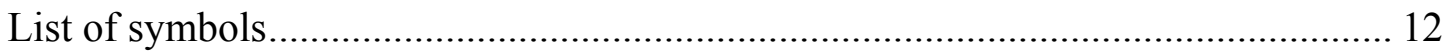

1 Introduction $\quad 14$

1.1 Concurrent research on CNTs and CNTs composites ………………............... 14

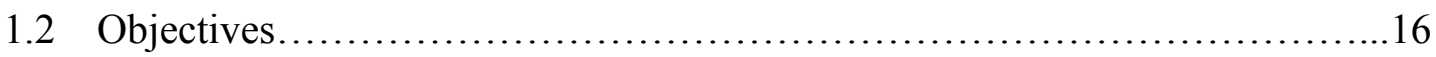

1.3 Thesis organization........................................................ 17

2 Literature review $\quad 19$

2.1 Introduction of CNTs and their composites...............................19

2.2 Mechanical properties of CNTs and their composites.........................22

2.3 Buckling behaviour of CNTs and their composites.........................22

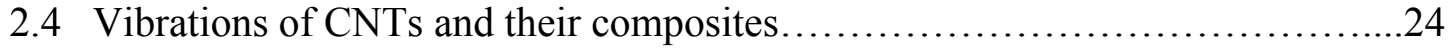




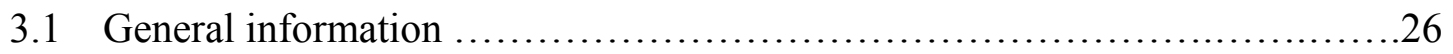

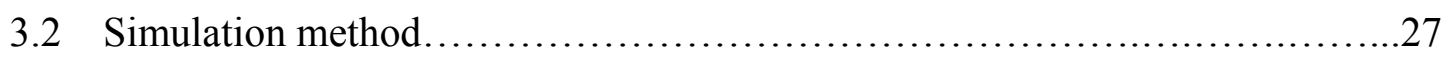

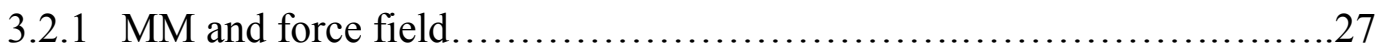

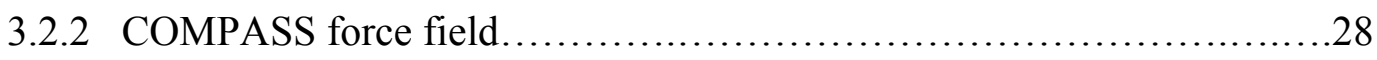

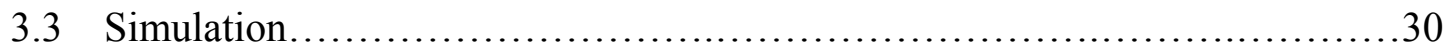

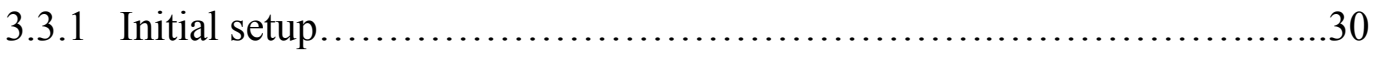

3.3.2 Minimizing setup.........................................................

3.3.3 Boundary condition setup.......................................... 34

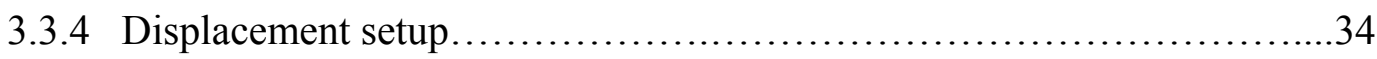

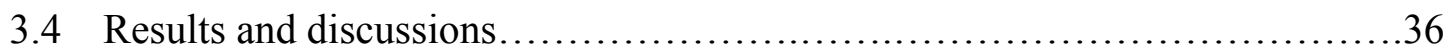

3.4.1 Buckling behaviour of PE-CNT matrices...............................36

3.4.2 The relations between the number of PE chains and the buckling

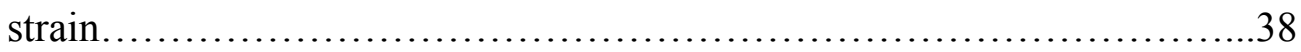

3.4.3 Factors that affect the results........................................ 41

4 VDW force between the PE chains and the CNT 47

4.1 General information......................................................

4.2 VDW force and Lennard-Jones potential.................................47

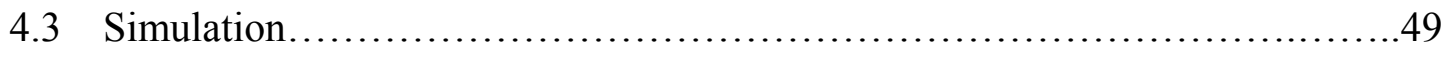

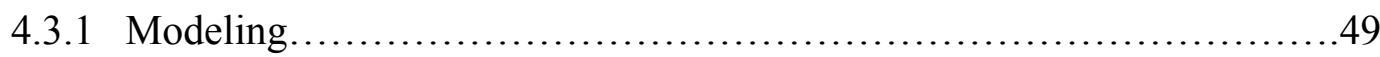

4.3.2 Simulation technique and process.................................49

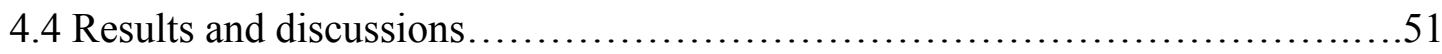


5 Vibrations of PE-CNT matrix

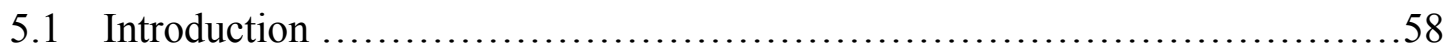

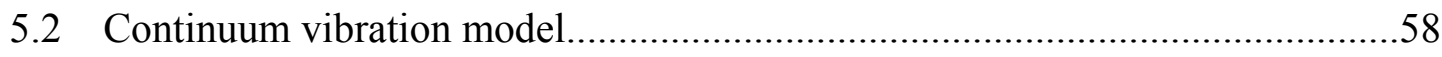

5.3 Simulation process..............................................62

5.3 .1 Modeling.......................................................62

5.3.2 Minimization and vibration analysis .............................62

5.4 Results and discussions...............................................63

6 Conclusion remarks and future work 66

6.1 Conclusion remarks.................................................66

6.2 Future work...................................................67

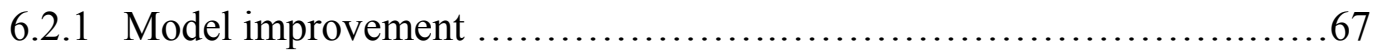

6.2.2 Beamlike buckling.........................................67

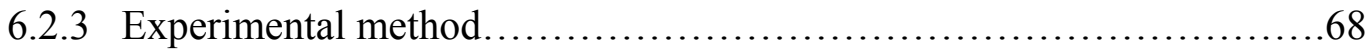

$\begin{array}{lr}\text { Back Matter } & 69\end{array}$

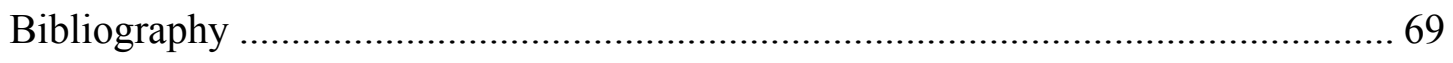




\section{List of Tables}

Table 1 Total strain energy VS displacement for 2x2x2 nm 15 PE chain matrix..........37

Table 2 (a) Buckling parameters for $2 \times 2 \times 2 \mathrm{~nm}$ matrices............................ 38

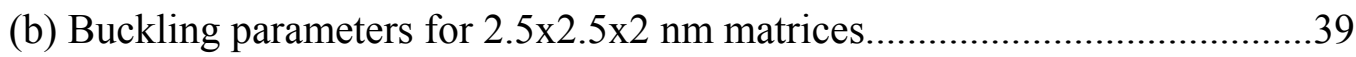

Table 3 The vdW potential for total, PE-PE, CNT-CNT, PE-CNT and the difference for

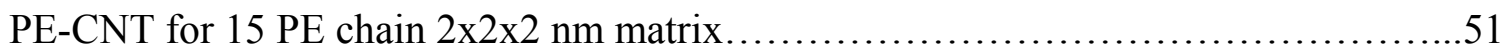

Table 4 Summary table parameters collected for $2 \times 2 \times 2 \mathrm{~nm}$ matrices.................53

Table 5 Table 5 Resonance frequencies for matrices containing 0, 1, 2, 4, 6, 8, 12 and 13 chains...................................................................... 64 


\section{List of Figures}

Figure 1 (a) The illustration of how a hexagonal sheet of graphite is "rolled" to form SWNTs of different chirality (b) Armchair, zigzag, and chiral SWNTs (from left to

right) .20

Figure 2 The off-axis and top views of MWCNTs with three walls

Figure 3 TEM micrographs of nanotube buckling in an aligned composite: (a) large-scale Euler-type buckling observed for small diameter carbon nanotubes, (b) local buckling/kinking of a large diameter carbon nanotube and (c) segmental buckling of a large diameter carbon nanotube at higher strain, indicating continued transfer of load to the nanotube after initial buckling. 24

Figure 4 Schematic representations of the radial breathing mode (RBM) and the G-band

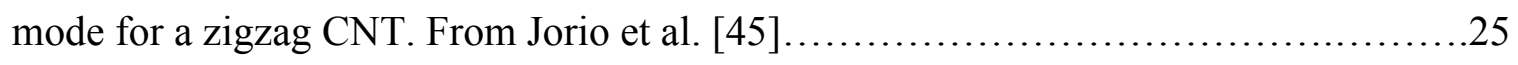

Figure 5 (a) Top view of CNT initially setup in $2 \times 2 \times 2 \mathrm{~nm}$ matrix (b) Side view of CNT initially setup in $2 \times 2 \times 2 \mathrm{~nm}$ matrix (c) Top view of CNT initially setup in $2.5 \times 2.5 \times 2 \mathrm{~nm}$ matrix (d) Side view of CNT initially setup in $2.5 \times 2.5 \times 2 \mathrm{~nm}$ matrix....................

Figure 6 (a) PE chain setup in $2 \times 2 \times 2 \mathrm{~nm}$ matrix (b) in $2.5 \times 2.5 \times 2.5 \mathrm{~nm}$ matrix..........32

Figure 7 (a) CNT, before adding constraints (b) CNT, after adding constraints..........34 
Figure 8 (a) Side view of displacement adding just before and (b) after buckling (c) Top view of displacement adding just before and (d) after buckling for $15 \mathrm{PE}$ chain $2 \times 2 \times 2 \mathrm{~nm}$ matrix. .35

Figure 9 (a) Top view of displacement adding just before and (b) after buckling for 24 $\mathrm{PE}$ chain $2 \times 2 \times 2 \mathrm{~nm}$ matrix .36

Figure 10 Total potential energy VS displacement for 2x2x2 nm 15 PE chain matrix....38 Figure 11 (a) One type of initial position for $20 \mathrm{PE}$ chain in $2.5 \times 2.5 \times 2 \mathrm{~nm}$ matrix before and (c) after minimizing (b) another type of initial position for 20 PE Chain in $2.5 \times 2.5 \times 2$ matrix before and (d) after minimizing. .42

Figure 12 For the $2.5 \times 2.5 \times 2 \mathrm{~nm}$ matrix (a) $28 \mathrm{PE}$ chain matrix and (b) $29 \mathrm{PE}$ chain matrix shape just after minimizing (c) 30 PE chain matrix under $1.1 \AA$ displacement and (d)

displacement. .43

Figure 13 A comparison of $2 \times 2 \times 2 \mathrm{~nm}$ PE-CNT matrix with (a) 10 chains and (b) 12 chains after minimizing. .44

Figure 14 (a) $15 \mathrm{PE}$ chain $2 \times 2 \times 2 \mathrm{~nm}$ matrix under $16 \AA$ displacement before (b) and after deleting the CNT.

Figure 15 The vdW potential for total, PE-PE, CNT-CNT, PE-CNT and the difference for PE-CNT for $15 \mathrm{PE}$ chain $2 \times 2 \times 2 \mathrm{~nm}$ matrix in curve. .52

Figure 16 (a) 16 PE Chain 2x2x2 matrix under $1.5 \AA$ (b) and $1.6 \AA$ displacement (c) 17 PE Chain 2x2x2 nm matrix under $1.4 \AA$ and $1.5 \AA$ displacement. .56 
Figure 17 Fundamental resonant frequencies from continuum shell model and MD simulations for clamped and cantilever SWCNTs of thickness $\mathrm{h}=0.34 \mathrm{~nm}$. $[59] \ldots \ldots . . .661$

Figure 18 (a) The shape of the matrix including 1 PE chain before (b) and after

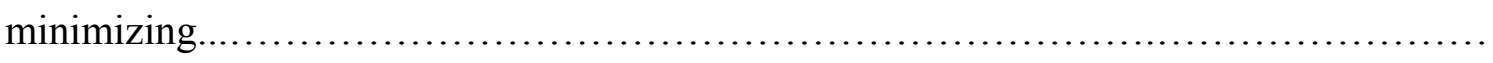
.......63 


\section{List of Abbreviations}

\begin{tabular}{|c|c|}
\hline CNT & Carbon nanotube \\
\hline SWCNT & Single wall carbon nanotube \\
\hline DWCNT & Double wall carbon nanotube \\
\hline MWCNT & Multi-wall carbon nanotube \\
\hline $\mathrm{PE}$ & Polyethylene \\
\hline $\mathrm{MD}$ & Molecular dynamics \\
\hline MM & Molecular mechanics \\
\hline VDW & Van der Waals \\
\hline $\mathrm{RBM}$ & Radial breathing mode \\
\hline MS & Materials Studio \\
\hline COMPASS & $\begin{array}{l}\text { Condensed-phase optimized molecular potentials for atomistic } \\
\text { simulation studies }\end{array}$ \\
\hline TEM & Transmission electron microscopy \\
\hline AFM & Atomic force microscopy \\
\hline PMMA & Poly (methyl methacrylate) \\
\hline CVD & Chemical vapor deposition \\
\hline
\end{tabular}




\title{
List of Symbols
}

\author{
$C_{h} \quad$ Chiral vector \\ $a_{1}, a_{2} \quad$ Unit vectors in two dimensional hexagonal lattice \\ $m, n \quad$ Translation indices \\ d Carbon nanotube diameter \\ $\theta \quad$ Chiral angle \\ E Effective Young's modulus beam material \\ I The moment of inertia of beam cross-section about its neutral axis \\ A Cross-sectional area of beam \\ $\rho \quad$ Mass density of beam material \\ w The transverse displacement of the beam \\ $f_{n} \quad$ Nth mode frequency \\ $L \quad$ Length of the beam \\ $k_{n} \quad$ Eigen value for $n$th mode \\ $p \quad$ Winkler-type elastic reaction force per unit length \\ $K \quad$ A constant depends on the material that the MWNT is embedded \\ $b, \theta, \phi, \chi \quad$ Internal coordinates of bond, angle, torsion angle, out-of-plane angle
}


$\delta_{i j} \quad$ Bond increments, represent the charge separation between two valencebonded atoms $i$ and $j$

$\varepsilon, r^{o} \quad$ Atomic partial charges and Lennard-Jones 9-6 (LJ-9-6) parameters

$j, i \quad j$ represents all atoms that are valence-bonded to atom $i$

$\mathrm{vdW}_{\text {Total }} \quad$ Total vdW potential between atoms in the matrix

vdW $\mathrm{PE-CNT} \quad \mathrm{vdW}$ potential between the atoms on PE chains and the atoms on CNTs

vdW $\quad$ ve-PE $\quad$ Potential between the atoms on PE chains

$\operatorname{vdW}_{\mathrm{CNT}-\mathrm{CNT}} \quad \mathrm{vdW}$ Potential between the atoms on CNT

$\Delta E \quad$ Difference of the vdW potential

$\Delta x \quad$ Average $\mathrm{x}, \mathrm{y}$ direction expansion displacement of a CNT under compression of $0.1 \AA$

[m], [c], [k] Mass, damping, and stiffness matrices

$\omega_{n} \quad$ Natural frequency 


\section{Chapter 1}

\section{Introduction}

\subsection{Concurrent research on CNTs and CNTs composites}

Since discovered by Iijima [1] in 1991, carbon nanotubes (CNTs) have generated great impact on science and engineering due to their outstanding physical and chemical properties.

CNTs are the strongest and stiffest materials. Overney et al. [2] calculated the rigidity of short SWNTs and found the Young's modulus to be $1500 \mathrm{GPa}$. A multi-walled CNT (MWCNT) was tested by Yu et al [3] and found to have a tensile strength of $63 \mathrm{GPa}$. The effects of the work function of the contact metal on the current-voltage characteristics of the CNT field-effect transistors (CNTFETs) have been studied by Mizutani et al. [4]. Researches on the thermal properties of nanotubes were carried out on ropes or bundles of tubes in early period of experimental work. Hone et al. [5] measured the thermal conductivity of a single wall CNT (SWCNT) mats at room temperature ranges from 2-35 $\mathrm{Wm}^{-1} \mathrm{~K}^{-1}$. Kim et al. [6] have used a microfabricated suspended device to measure the 
thermal conductivity of MWCNTs and obtained the thermal conductivity over $3000 \mathrm{~W} / \mathrm{K}$ at room temperature.

Nowadays, mechanical behaviours of CNTs subjected to axial loading have drawn attention. Buckling is a very important research topic that has been studied by both molecular mechanics method (MM) and experimental method. Yakobson et al. [7] first employed molecular dynamics (MD) simulations and continuum mechanics models on the buckling of SWCNTs under axial compression. By employing MD simulations, Liew et al. [8] studied the buckling behaviour of axially compressed MWCNTs. Iijima et al. [9] utilized high resolution electron microscopes to observe the CNTs buckling behaviour under bending and explained by MD simulations. Moreover, Wang et al. [10] studied the local buckling of CNTs under bending as well as the torsional buckling of double wall CNT (DWCNTs) [11] using MM simulation.

Concurrently, CNTs are considered ideal candidates as filler materials in composites. Kilbride et al. [12] measured both alternating current (ac) and direct current (dc) in polymer-nanotube composite thin films. Biercuk et al. [13] found that the thermal conductivity of the composite is higher than the polymer itself by adding SWCNTs to epoxy. To the author's acknowledgement, researching on buckling behaviour of CNT composites is very essential; and in this case, to obtain the relation between the buckling behaviour of CNTs and CNTs composites becomes a key point of the research. Researches on buckling behaviour of CNT composite have been done by both MM method and experimental method. Wang [14] studied the buckling behaviour of single polyethylene molecule (PE) wrapped CNT and concluded that the van der Waals (vdW) interaction induced a decrease in buckling strain. Thostenson and Chou [15] studied the 
nanotube buckling in aligned MWCNT composites using transmission electron microscopy (TEM) observation.

Besides the buckling behavior, the dynamic characteristics are also significant in the applications in $\mathrm{CNT} /$ polymer composites. It is reported that significant increases in internal damping of polymer nanocomposites when CNTs are used as reinforcement. Koratkar et al. [16, 17] reported that densely packed MWNT nano-films have been embedded as interlayers within laminated piezo-silica sandwich beams to enhance the flexural stiffness by $30 \%$ and damping of the laminates by $100 \%$. Moreover, loads applied on the composites result in strains of the nanotubes can be detected as changes of the carbon vibration frequencies using Raman spectroscopy [18].

\subsection{Objectives}

First, in order to find a design of high strength polyethylene-CNT (PE-CNT) matrix with proper PE/CNT ratio, the buckling strain of PE-CNTs polymer matrices with different number of PE chains will be calculated using MM simulation. Subsequently, the buckling strain of the SWCNT and PE-CNTs polymer matrix will be compared.

Second, the vdW interaction between PE chains and SWCNT before buckling will be studied using MM simulation, which may provide an explanation for the buckling behaviour.

Third, the vibration of PE-CNT matrices will be analyzed and compared to the vibration of pristine SWCNTs in order to discover their dynamic characteristics. 


\subsection{Thesis organization}

This thesis contains 6 chapters. These chapters are organized are as follows:

Chapter 1-Introduction: This chapter provides general information on concurrent research on CNTs and CNTs composites, the objective of this thesis, and the thesis organization

Chapter 2-Literature review of mechanical properties of CNTs and their composites: This part first gives a brief introduction of CNTs and their composites; then emphasize on the mechanical properties, concurrent research on buckling and vibrations of CNTs and their composites.

Chapter 3-Buckling behaviour of PE-CNT matrix composite: This chapter uses MM simulation method to find the buckling strain for different PE chains with SWCNTs both in $2 \times 2 \times 2 \mathrm{~nm}$ matrix and $2.5 \times 2.5 \times 2 \mathrm{~nm}$ matrix and discusses the relation of the number of PE chains and the buckling strain.

Chapter 4-VDW force between PE chains and CNT: This chapter analyzes the vdW force among PE chains and CNTs before buckling for $2 \times 2 \times 2 \mathrm{~nm}$ matrix and finds that the vdW force will provide strong effect on the buckling strain in chapter 3.

Chapter 5-Vibrational modes of PE-CNTs matrix composite: This chapter analyzes the different "fundamental" modes for $2 \times 2 \times 2 \mathrm{~nm}$ PE-CNT matrix composite and provides the relation of the number of PE chains and the vibrational frequencies. This result also provides relations on the number of PE chains and the buckling behaviour in chapter 3 . 
Chapter6 - Concluding remarks and future work: This part is generally the conclusion of the thesis as well as indicating the future job. 


\section{Chapter 2}

\section{Literature review}

\subsection{Introduction of CNTs and their composites}

CNTs, discovered by Iijima [1,19], are tubular carbon molecules whose lengths range from tens nanometers up to several microns [20-23]. CNTs own significant mechanical, electronic and thermal properties and own significant capacity for applications in nanocomposites, nanodevices, and nanoelectronics [24-27]. It is expected that CNTs will have great impacts on industries from medicine, agriculture, environment, and biotechnology to information technology, aerospace, and energy. The two main types of CNTs are SWNT $[1,19,20]$, which is a seamlessly rolled single sheet of graphene cylinder formed with diameter of order of $1 \mathrm{~nm}$ and length of up to centimetres and MWCNT which consist of an array of such cylinders formed concentrically. SWCNTs are considered as a graphene sheet rolled up along its chiral vector $C_{h}=m a_{1}+n a_{2}$ as shown in Fig. 1(a), where $a_{1}$ and $a_{2}$ are unit vectors in the two-dimensional hexagonal lattice and the integers $m$ and $n$ are the translation indices. The perimeter of the SWCNT 
$(m, n)$, formed along $C_{h}$, is equal to the length of the vector. The diameter $d$ of the $\operatorname{SWCNT}(m, n)$ is then calculated as

$$
d=\frac{\left|\overline{C_{h}}\right|}{\pi}=0.0783 \sqrt{n^{2}+n m+m^{2}} \mathrm{~nm}
$$

and the chiral angle $\theta$ between $C_{h}$ and $a_{1}$ is given by

$$
\theta=\sin ^{-1}\left[\frac{\sqrt{3} m}{2 \sqrt{n^{2}+n m+m^{2}}}\right]
$$

which ranges from $0^{\circ}$ to $30^{\circ}$ owing to the hexagonal symmetry of the carbon atoms. Different types of SWCNTs are, thus, uniquely defined by the integer pair $(m, n)$ or the corresponding chiral angle $\theta$.

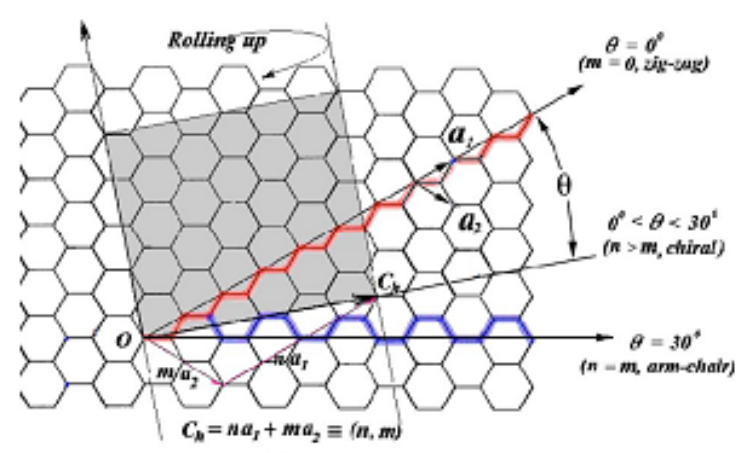

(a)
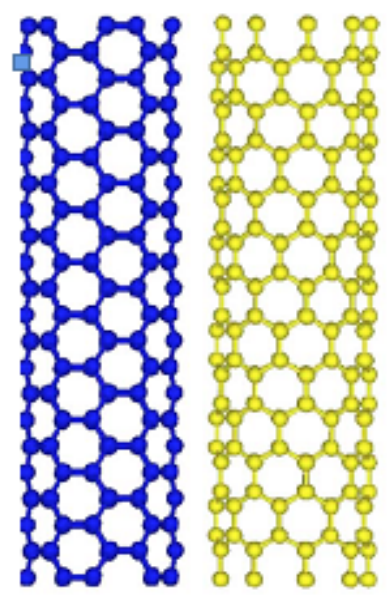

(b)

Figure 1 (a) The illustration of how a hexagonal sheet of graphite is "rolled" to form SWNTs of different chirality. (b) Armchair, zigzag, and chiral SWNTs [28] (from left to right) 
In literature, SWCNTs have been classified into three categories, i.e., (1) zigzag CNTs with $\theta=0^{\circ}(m=0)$, (2) chiral CNTs with $0^{\circ}<\theta<30^{\circ}$ (where $m>n$ ), and (3) armchair CNTs with $\theta=30^{\circ}$ (where $m=n$ ). These three different SWCNTs are illustrated in Fig. 1(b). MWCNTs comprise two or more concentric SWCNTs with usually different chiral angles as shown in Figure $2[1,23,25,29]$. The constituent SWCNTs are nested within one another, with the interlayer spacing of $0.34 \mathrm{~nm}$, close to $0.335 \mathrm{~nm}$ for the graphite layers [30-32]. The constituent SWNCTs interact with each other via the vdW forces.
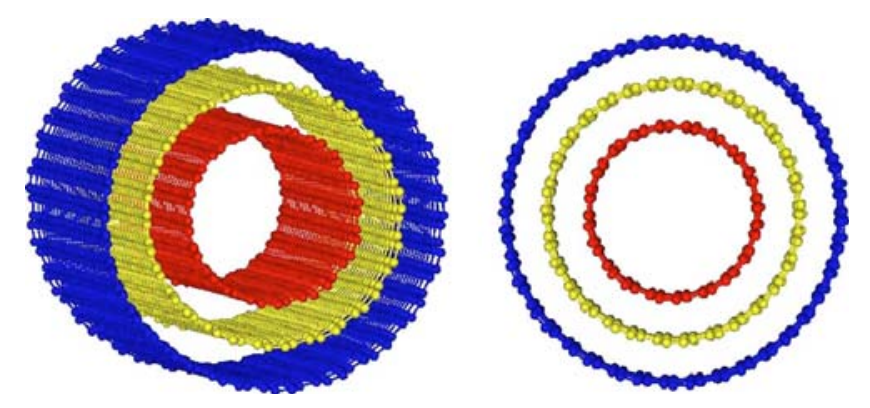

Figure 2 The off-axis and top views of MWCNTs with three walls [28]

The combination of superlative mechanical, thermal and electronic properties of CNTs makes them ideal candidates as advanced filler materials in composites. However, probably the most promising area of composites research involves the mechanical enhancement of plastics using CNTs as reinforcing fillers [33]. 


\subsection{Mechanical properties of CNTs and their composites}

$\mathrm{Lu}$ [34] predicted that the Young's modulus of CNTs was close to $1 \mathrm{TPa}$ independent of nanotube type and diameter using an empirical simulation model. Treacy et al. [35] estimated Young's modulus of isolated CNTs by measuring, in the transmission electron microscope, the amplitude of their intrinsic thermal vibrations and calculated the moduli of 0.40-4.15 TPa for different numbers of tubes, which is the first actual mechanical measurement. The atomic force microscope (AFM) is applied for the first direct measurement to obtain the stiffness constant of arc-MWCNTs for one end fixed and the average Young's modulus is found to be 1.28 TPa by Wong et al. [36] in 1997. Jin et al. [37] worked on dynamic mechanical behaviour on CNT/poly(methyl methacrylate) composite. They tested PMMA doped with Arc-MWNT by DMA and found that the Young's modulus increased from $0.7 \mathrm{GPa}$ to $1.63 \mathrm{GPa}$ at $17 \mathrm{wt} . \% \mathrm{CNTs}$. Studies on CVD-MWNT in polystyrene $[38,39]$ reported that the modulus increased from 2 GPa to $2.6 \mathrm{GPa}$ and $4.5 \mathrm{GPa}$ at $5 \mathrm{wt} . \%$ and 25 vol.\%, respectively, providing reinforcement levels of 9 and $19 \mathrm{GPa}$.

\subsection{Buckling behaviour of CNTs and their composites}

Tremendous efforts have been put on the buckling analysis of CNTs under diverse loading conditions. In this section, the buckling behaviour due to axial loading under compression is discussed.

The first detailed investigation on the buckling of SWCNTs under axial compression was done by Yakobson et al. [7] who employed MD simulations and explained by continuum 
mechanics models. It was observed that a SWCNT with an aspect ratio of 6 subject to large deformations reversibly switch into different morphological patterns. For such SWCNT with a relatively small aspect ratio, shell-buckling mode is observed. On the other hand, for a slender SWCNT with a large aspect ratio, the Euler beam buckling mode is observed instead. Thus, CNTs under axial compression demonstrate similar buckling modes to those predicted by continuum mechanics models.

Despite the extensive atomistic simulations on CNT buckling, very few experimental results exist. From the few sample results, Lourie et al. [40] and Bower et al. [41] employed TEM to obtain Euler-beam buckling modes of MWCNTs. Waters et al. [42] obtained experimental results of shell buckling instabilities in vertically aligned MWCNTs under uniaxial compression. The experimental observations were found to agree well with the predictions of linear elastic shell buckling theory. Later, Waters et al. [43] conducted experiments on individual MWCNTs under axial compression in order to measure the critical shell-buckling load.

Very few researches have been conducted on buckling behaviour of CNTs composites. In 2004, Thostenson et. al [44] observed compressive buckling of aligned MWCNTPolystyrene Composite matrix through TEM and the graph is shown in Figure 3. 

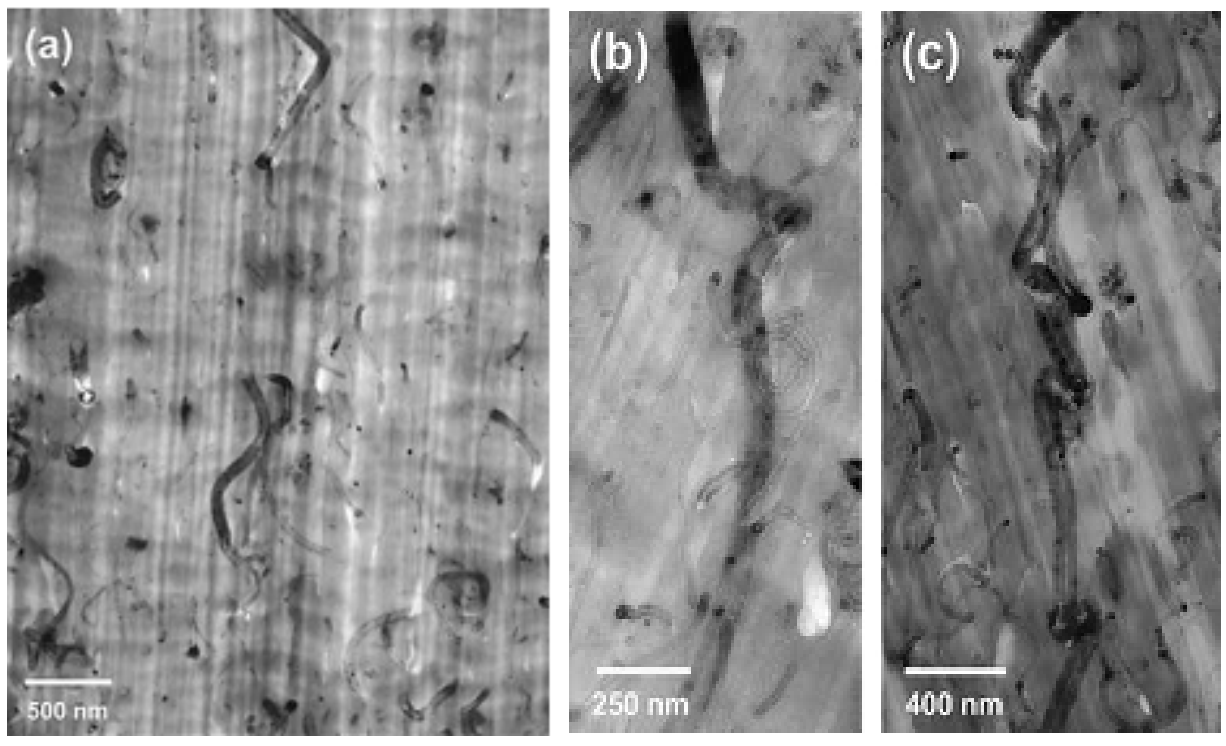

Figure 3 TEM micrographs of CNT buckling in an aligned composite: (a) large-scale Euler-type buckling observed for small diameter CNTs, (b) local buckling/kinking of a large diameter $\mathrm{CNT}$ and (c) segmental buckling of a large diameter CNT at higher strain, indicating continued transfer of load to the CNT after initial buckling.

\subsection{Vibrations of CNTs and their composites}

The one-dimensional nature of CNTs has pronounced effects on their vibrational properties. Spectroscopic work on the experimentally observable quantities of the tubes has become extremely rewarding as regards fundamental insight as well as the information used to characterize CNTs. It turns out that for the largest part of the vibrational properties combining CNT symmetry and electronic band structure is essential to gain deeper insight. Raman spectroscopy is a widely used technique in materials science which involves the excitation of a sample with intense monochromatic 
light (typically laser light) followed by observations of the scattering of the incident radiation and it is used extensively to study vibrational modes of CNTs. The basic concepts and characteristics of Raman spectra from CNTs have been summarized by Ecklund et al. [45], Jorio et al. [46] and Dresselhaus et al. [47]. A review article on Raman spectroscopy of individual SWNTs has been published by Dresselhaus et al. [48]. The radial breathing mode (RBM) and the G-band mode, which are two of many possible modes of vibration of the atoms in a CNT, are shown schematically in Figure 4.
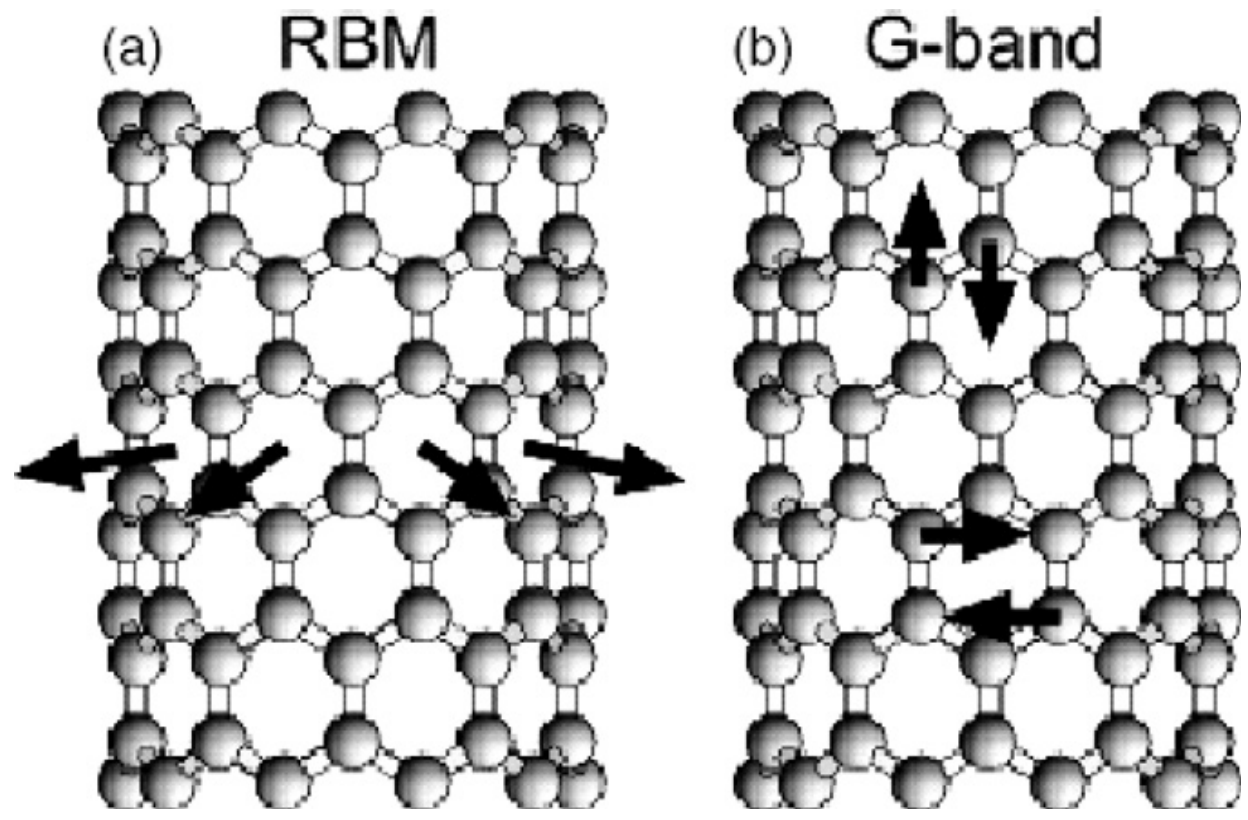

Figure 4 Schematic representations of the radial breathing mode (RBM) and the G-band mode for a zigzag CNT [46]. 


\section{Chapter 3}

\section{Buckling behaviour of PE-CNT composites}

\subsection{General information}

Although the buckling behaviour of CNTs was studied by many researchers, little research has been done on the buckling behaviour of CNTs composites. Moreover, to the author's knowledge, no studies, whether through experiments or simulations, have been published on buckling behaviour of PE-CNTs. The purpose of this chapter is not only to find the buckling strain of the composite, but also to find how the buckling strain changes as the density of the matrix increases (i.e. how the buckling strain changes with the

number of PE chains increases). The key point of this work is to make appropriate assumptions and set up proper simulation methods, and finally analyze the simulation results.

\subsection{Simulation method}


Materials Studio 4.0 (Accelrys) [49] is employed to calculate the energies such as total potential energy, internal energy, vdW potential, etc.

\subsubsection{MM and force field}

The interactions between atoms are the foundations of molecular simulation, which include the interactions of chemical bond between atoms as well as the vdW interactions between molecules or hydrogen bond. There are two ways of describing these interactions: One way is chemical calculation and the other way is to employ the force field. MM uses Newtonian mechanics to model molecular systems. The potential energy of all systems in MM is calculated using force field.

Force-field development, as a fundamental issue underlying all atomistic simulations, has drawn considerable attention in recent years. To construct a force field generally suitable for condensed phase applications, it was necessary to modify the nonbond parameters, and consequently, the valence parameters must also be changed due to the coupling between the valence and nonbond parameters. Basically, a hybrid approach consisting of both ab initio and empirical methods was employed to derive a new general force field based on the PCFF force field. In addition to those molecular classes covered in the PCFF force field, a number of new molecular classes were parameterized. Most significantly, nonbond parameters were completely re-parameterized. The outcome is a new, condensed phase-optimized ab initio force field. This force field is named COMPASS (condensed-phase optimized molecular potentials for atomistic simulation studies).

\subsubsection{COMPASS force field}


The functional forms used in this force field are the same as those used in CFF-type force fields

$$
\begin{aligned}
& E_{\text {total }}=\sum_{b}\left[k_{2}\left(b-b_{o}\right)^{2}+k_{3}\left(b-b_{o}\right)^{3}+k_{4}\left(b-b_{o}\right)^{4}\right]+ \\
& \sum_{\theta}\left[k_{2}\left(\theta-\theta_{o}\right)^{2}+k_{3}\left(\theta-\theta_{o}\right)^{3}+k_{4}\left(\theta-\theta_{o}\right)^{4}\right]+ \\
& \sum_{\phi}\left[k_{1}(1-\cos \phi)+k_{2}(1-\cos 2 \phi)+k_{3}(1-\cos 3 \phi)\right]+ \\
& \sum_{\chi} k_{2} \chi^{2}+\sum_{b, b^{\prime}} k\left(b-b_{o}\right)\left(b^{\prime}-b_{o}^{\prime}\right)+\sum_{b, \theta} k\left(b-b_{o}\right)\left(\theta-\theta_{o}\right)+ \\
& \sum_{b, \phi}\left(b-b_{o}\right)\left[k_{1} \cos \phi+k_{2} \cos 2 \phi+k_{3} \cos 3 \phi\right]+ \\
& \sum_{\theta, \phi}\left(\theta-\theta_{o}\right)\left[k_{1} \cos \phi+k_{2} \cos 2 \phi+k_{3} \cos 3 \phi\right]+ \\
& \sum_{b, \theta} k\left(\theta^{\prime}-\theta_{o}^{\prime}\right)\left(\theta-\theta_{o}\right)+\sum_{\theta, \theta^{\prime}, \phi} k\left(\theta-\theta_{o}\right)\left(\theta^{\prime}-\theta_{o}^{\prime}\right) \cos \phi+ \\
& \sum_{i, j} \varepsilon_{i j}\left[2\left(\frac{r_{i j}^{o}}{r_{i j}}\right)^{9}-3\left(\frac{r_{i j}^{o}}{r_{i j}}\right)^{9}\right]
\end{aligned}
$$

The functions can be divided into two categories valence terms including diagonal and off-diagonal cross-coupling terms and nonbond interaction terms. The valence terms represent internal coordinates of bond $(b)$, angle $(\theta)$, torsion angle $(\phi)$, and out-ofplane angle $(\chi)$, and the cross-coupling terms include combinations of two or three internal coordinates. The crosscoupling terms are important for predicting vibration frequencies and structural variations associated with conformational changes. Among the cross-coupling terms given in equation 3.1, the bond-bond, bond-angle and bond-torsion angle are the most frequently used terms. The nonbond interactions, which include a LJ9-6 function for the vdW term and a Coulombic function for an electrostatic interaction, 
are used for interactions between pairs of atoms that are separated by two or more intervening atoms or those that belong to different molecules. In comparison with the common LJ-12-6 function, which is known to be too 'hard' in the repulsion region, the LJ-9-6 function is softer but may be too attractive in the long separation range. However, this difference appears to be unimportant to the properties of interest in this work based on a comparative study carried out at the beginning of this project. The LJ-9-6 parameters ( $\varepsilon$ and $r^{o}$ ) are given for like atom pairs. For unlike atom pairs, a 6th order combination law is used to calculate the off-diagonal parameters:

$$
\begin{aligned}
& r_{i, j}=\left(\frac{\left(r_{i}^{o}\right)^{6}+\left(r_{j}^{o}\right)^{6}}{2}\right)^{1 / 6} \\
& \varepsilon_{i, j}=2 \sqrt{\varepsilon_{i} \varepsilon_{j}}\left(\frac{\left(r_{i}^{o}\right)^{3} \cdot\left(r_{j}^{o}\right)^{3}}{\left(r_{i}^{o}\right)^{6} \cdot\left(r_{j}^{o}\right)^{6}}\right)
\end{aligned}
$$

The electrostatic interaction is represented using atomic partial charges. To make the charge parameters transferable, bond increments $\delta_{i j}$, which represent the charge separation between two valence-bonded atoms $i$ and $j$, are used in the force field as parameters. For atom $i$, the partial charge is the sum of all charge bond increments $\delta_{i j}$

$$
q_{i}=\sum_{j} \delta_{i j}
$$

where $j$ represents all atoms that are valence-bonded to atom $i$.

In condensed-phase simulations of liquids and crystals, the nonbond interactions are usually truncated at a selected cutoff value (normally around $10 \AA$ ). A sharp cutoff is assumed for the present force field. However, the long-range interaction, which is the 
total contribution of nonbond interactions beyond the cutoff, is critically important to be considered for calculating energies and pressures. [50]

COMPASS is a powerful forcefield supporting atomistic simulations of condensed phase materials. COMPASS stands for condensed-phase optimized molecular potentials for atomistic simulation studies. COMPASS is the first ab initio forcefield that has been parameterized and validated using condensed-phase properties, in addition to various ab initio and empirical data for molecules in isolation. Consequently, this forcefield enables accurate and simultaneous prediction of structural, conformational, vibrational, and thermophysical properties for a broad range of molecules in isolation and in condensed phases, and under a wide range of conditions of temperature and pressure. The most recent enhancements to the COMPASS forcefield have concentrated on parameterization of more than 45 inorganic oxide materials and mixed systems, including interfaces of organic and inorganic materials. [51]

\subsection{Simulation}

\subsubsection{Initial setup}

\section{Matrix setup and matrix dimensions}

The nano-scale system is composed of a CNT and a PE matrix. The dimensions of the matrix are $2 \times 2 \times 2 \mathrm{~nm}$ and $2.5 \times 2.5 \times 2 \mathrm{~nm}$. The matrices are replicated across periodic boundaries in all 3 dimensions which will make the SWCNT and PE chains infinitely long. The whole simulation process is done using Materials Studio 4.0 (Accelrys). 


\section{CNT parameters}

A $(5,5)$ SWCNT with 8 repeated units is embedded in a crystalline polymer matrix, whose diameter is $6.78 \AA$ and length is $19.68 \AA$. The CNT is initially located at the center of the matrix. The shape and position of the CNT are shown in Figure 5 (a), (b), (c) and (d). Although the CNTs in the matrices have the same size, the different dimensions of the matrices show that the CNT in the $2.5 \times 2.5 \times 2 \mathrm{~nm}$ matrix looks much smaller from the top view and much thinner from the side view.

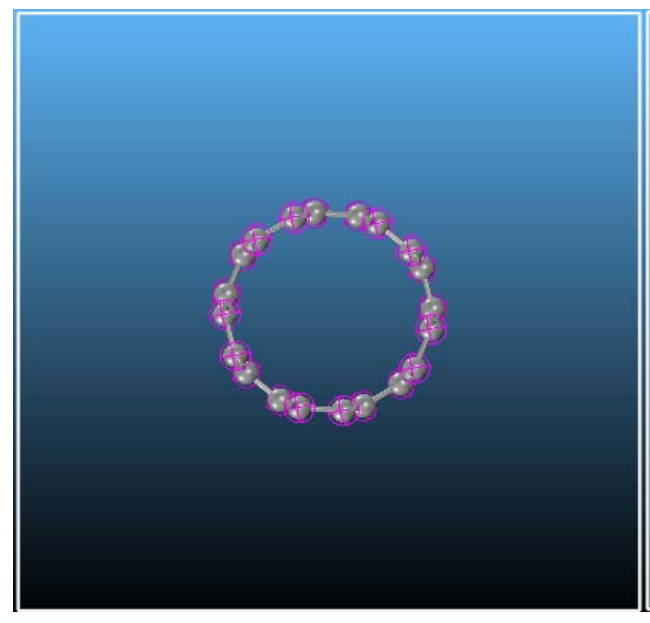

(a)

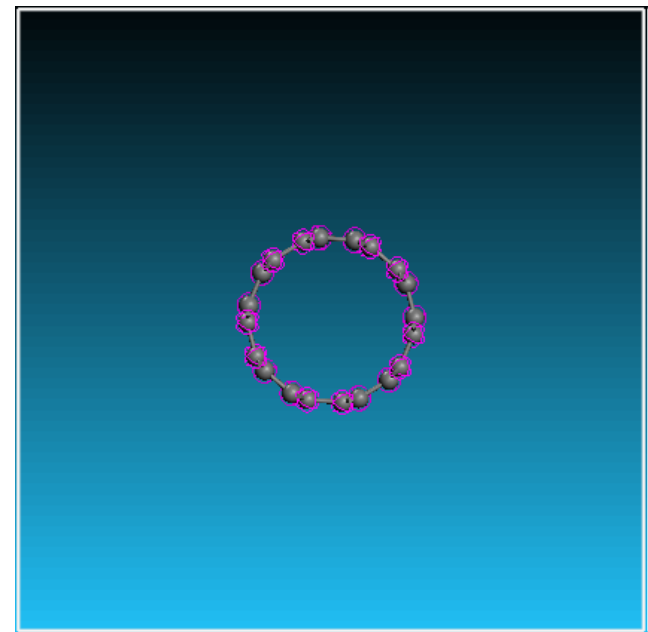

(c)

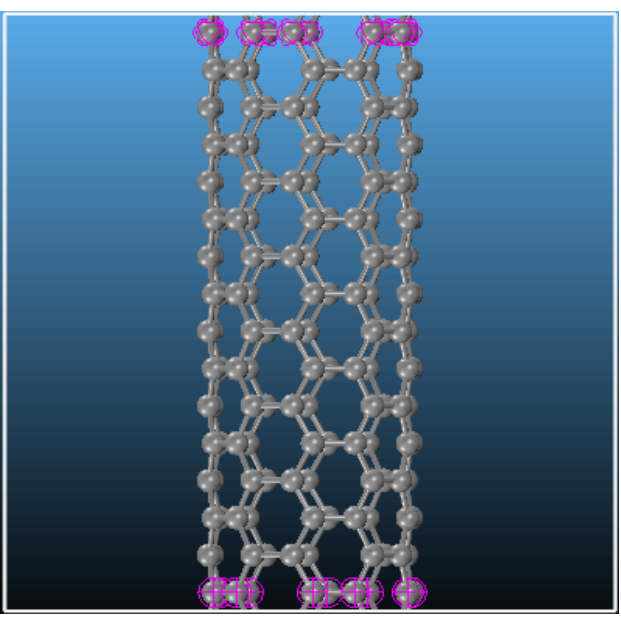

(b)

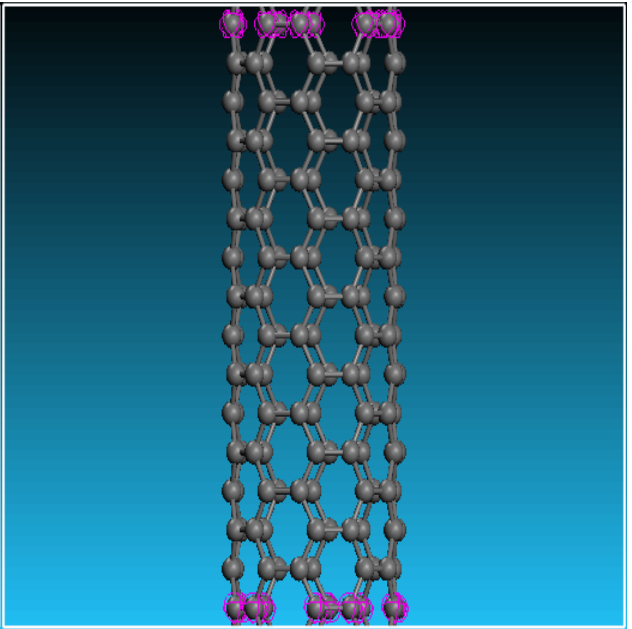

(d) 
Figure 5 (a) Top view of CNT initially setup in $2 \times 2 \times 2 \mathrm{~nm}$ matrix (b) Side view of CNT initially setup in $2 \times 2 \times 2 \mathrm{~nm}$ matrix (c) Top view of CNT initially setup in $2.5 \times 2.5 \times 2 \mathrm{~nm}$ matrix (d) Side view of CNT initially setup in $2.5 \times 2.5 \times 2 \mathrm{~nm}$ matrix

\section{PE chain numbers and parameters}

One typical model for the $2 \times 2 \times 2 \mathrm{~nm}$ matrix contains 15 chains of 16 methylene units and 2 methyl groups on the ends with the hydrogen atoms. The $15 \mathrm{PE}$ chains are initially set up around the CNT. The shape of the 15 PE chain matrix looks like an "imperfect" square. The initial setup distance between every two adjacent PE chains is $3.5 \AA$, along both the horizontal and vertical directions. The buckling behaviours of matrices filled with 11-18 chains are all set up and compared.

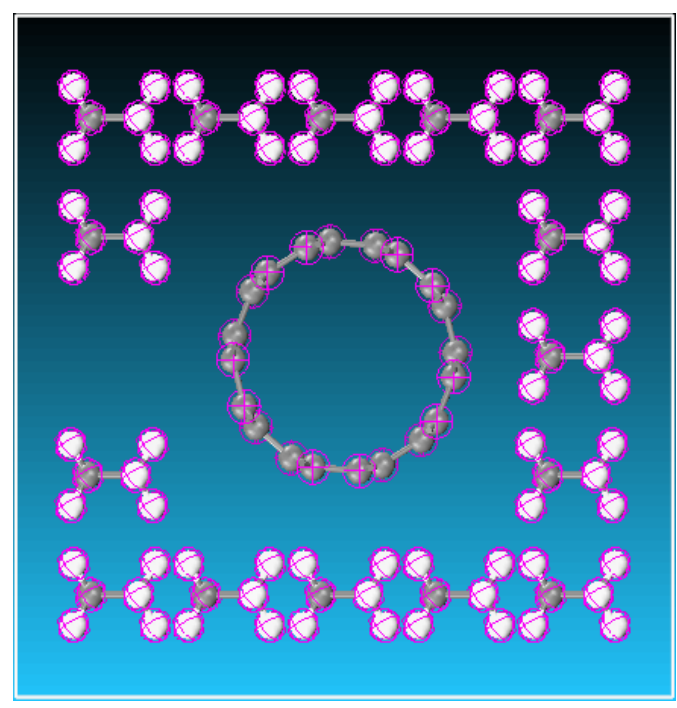

(a)

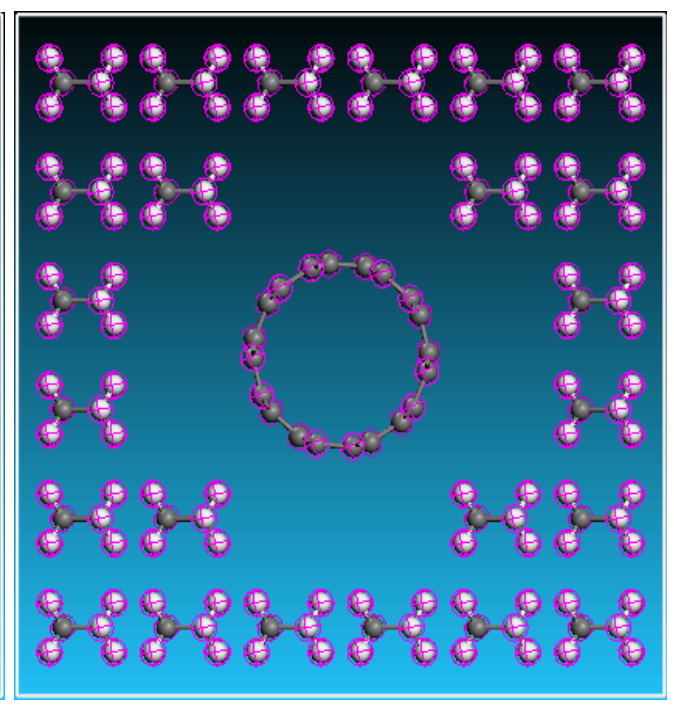

(b)

Figure 6 (a) PE chain setup in $2 \times 2 \times 2 \mathrm{~nm}$ matrix (b) in $2.5 \times 2.5 \times 2.5 \mathrm{~nm}$ matrix 
One typical model for the $2.5 \times 2.5 \times 2 \mathrm{~nm}$ matrix contains 24 chains of 16 methylene units and 2 methyl groups on the ends with the hydrogen atoms. The initial setup distance between every two adjacent PE chains is $4.0 \AA$, along both the horizontal and vertical directions. The buckling behaviours of matrices filled with 22-29 chains are all set up and compared. Figure 6 (a) and (b) show the details of PE chains set up in $2 \times 2 \times 2 \mathrm{~nm}$ and $2.5 \times 2.5 \times 2.5 \mathrm{~nm}$ matrices.

\subsubsection{Minimizing setup}

The minimization process is the simulation of the real PE-CNT synthesis process. By employing the COMPASS force field, a list of parameters is calculated using the software in a list called minimization energy summary. The procedure is as follows: First, set " compass" as the force field from tab "Discover Setup"; next, on the "Job control" tab, select "Run in parallel 4 of 4 processors" to make the calculation as fast as possible. Then click on "Discover Minimizer" and use "Smart Minimizer". Finally, customize the maximum iteration steps to be 300000 . Using such a big number ensures a complete minimization process.

After finishing the calculation step, the minimizing energy summary list is shown. The first minimizing process is done before the boundary condition since it indicates the simulation of PE-CNT synthesis, which is the first step after setting up the initial position of the PE chains and the CNT. However, if we are eager to see the buckling behaviour, displacements must be applied axially to the CNT, which essentially needs a boundary condition setup. 


\subsubsection{Boundary condition setup}

Constraints are added to the top and bottom portions of the CNT. The constraints are added on all 3 dimensions. The details of adding constraints to the CNT are shown in Figure 7 (a) and (b).

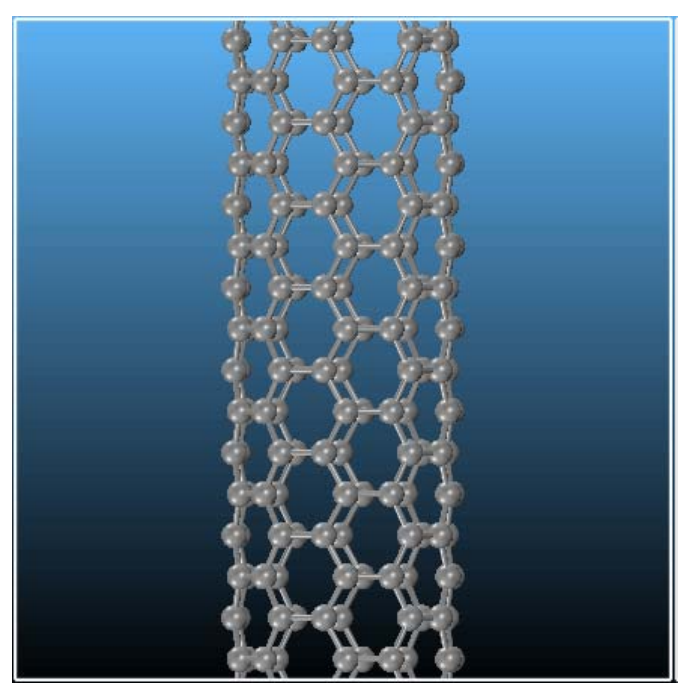

(a)

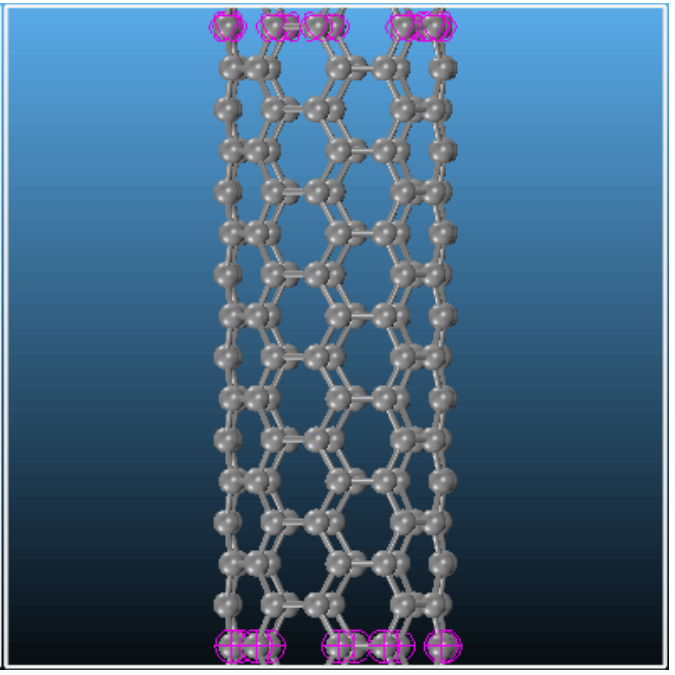

(b)

Figure 7 (a) CNT, before adding constraints (b) CNT, after adding constraints

\subsubsection{Displacement setup}

After adding constraints to the CNT, axial displacement will be added to both top and bottom portions of the CNT at the same distance. The distance is set at $0.1 \AA$ for a total of $0.05 \AA$ on each side every time. After each displacement, Smart minimizer is employed 
to calculate the energy of each step. The displacements are added gradually until the CNT buckles. Figure 8 (a) and (b) shows the side view of the displacement just before and after buckling for $15 \mathrm{PE}$ chain $2 \times 2 \times 2 \mathrm{~nm}$ matrices. For easy visualization, the PE chains are cleared. Figure 8 (c) and (d) show the top view of 15 PE chain $2 \times 2 \times 2 \mathrm{~nm}$ matrices just before and after buckling.

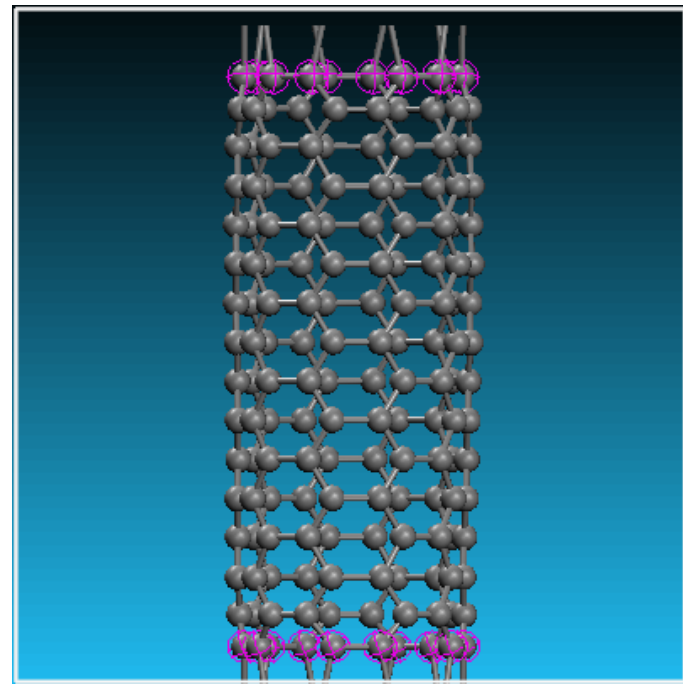

(a)

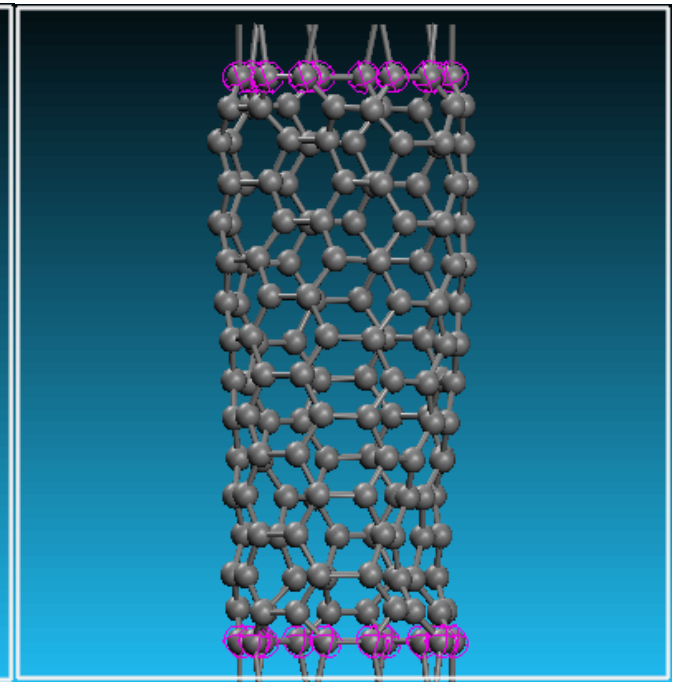

(b)

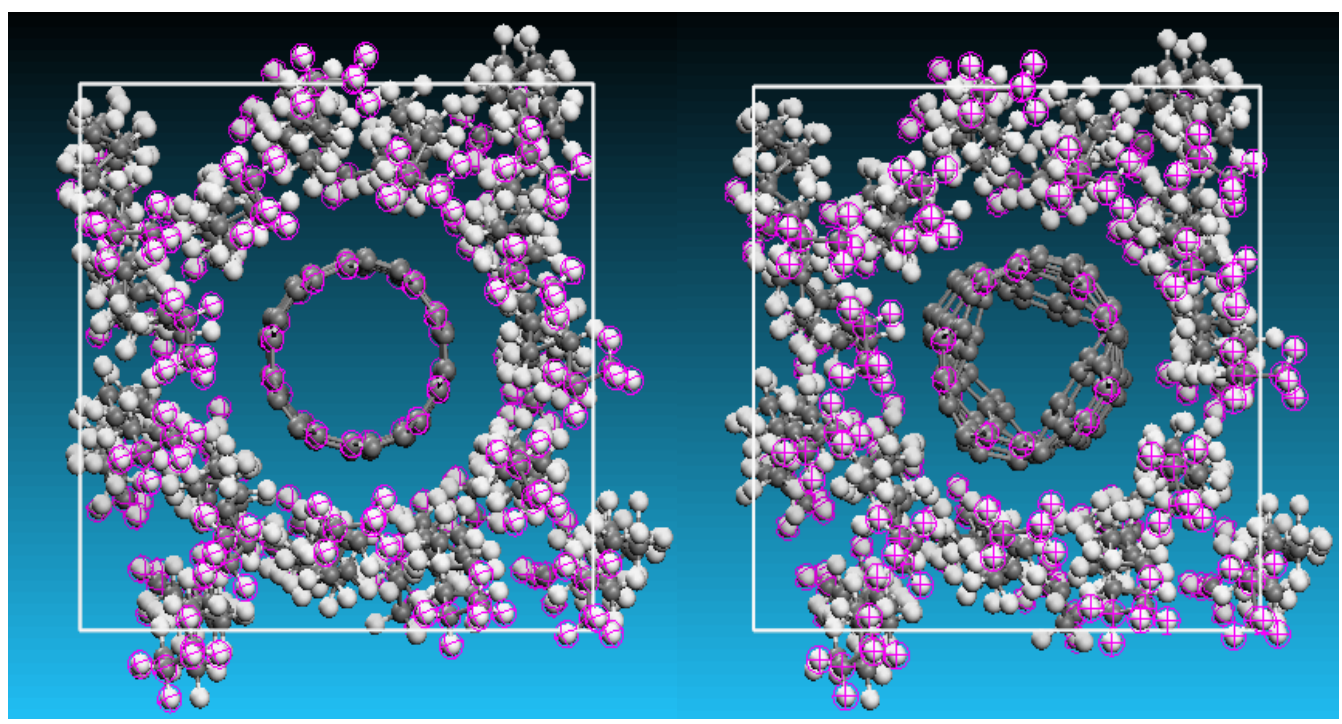


Figure 8 (a) Side view of displacement added just before and (b) after buckling (c) Top view of displacement added just before and (d) after buckling for the 15 PE chain $2 \times 2 \times 2$ nm matrix.

\subsection{Results and discussions}

\subsubsection{Buckling behaviour of PE-CNT matrices}

As visualized in Figure 5 (c) and (d), it is found that by adding a displacement of $1.9 \AA$, the $2 \times 2 \times 2 \mathrm{~nm}$ matrix with $15 \mathrm{PE}$ chains starts to buckle. In other words, the buckling strain is $10.86 \%$, which is the ratio of displacement to the original CNT length in the matrix (17.500 $\AA$ ). Similarly, from Figure 9 (a) and (b), we found that by adding a displacement of $2.1 \AA$, the $2.5 \times 2.5 \times 2 \mathrm{~nm}$ matrix with $24 \mathrm{PE}$ chains starts to buckle, and the buckling strain is measured at $12.00 \%$.

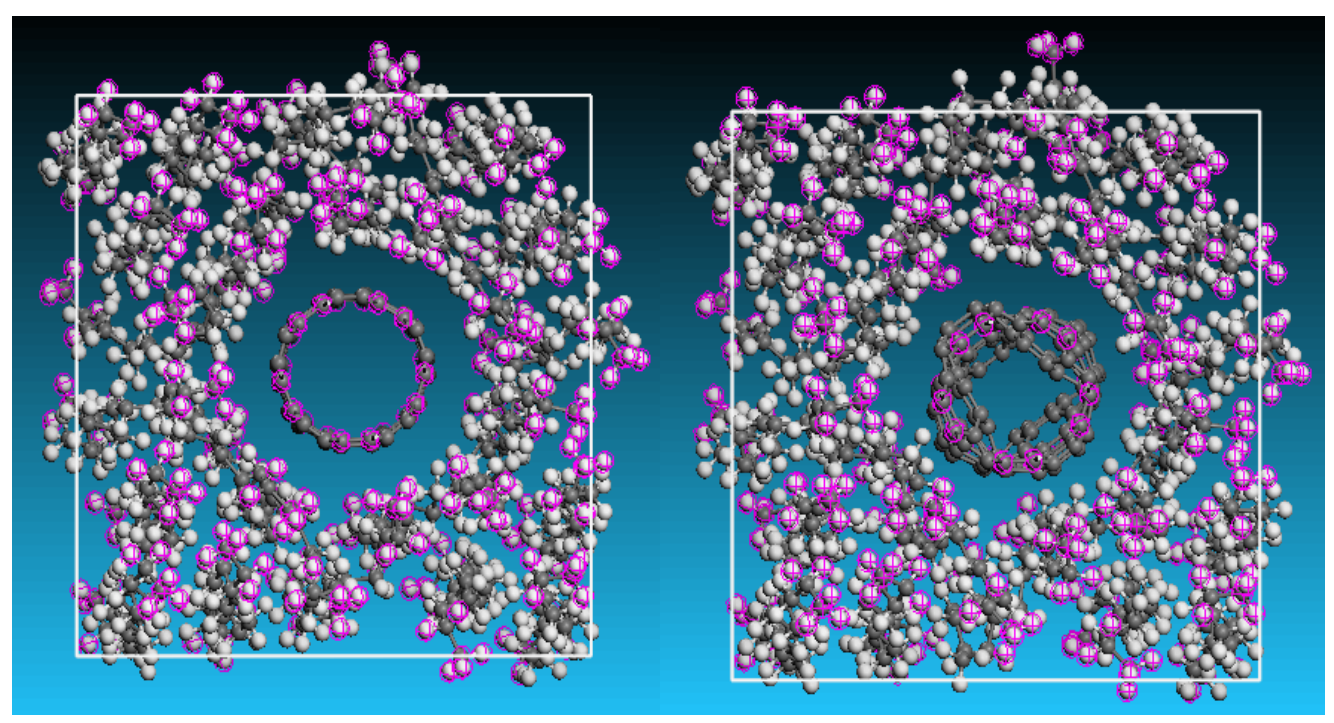


Figure 9 (a) Top view of displacement added just before and (b) after buckling for $24 \mathrm{PE}$ chain $2 \times 2 \times 2 \mathrm{~nm}$ matrix.

The occurrence of buckling can also be defined as a spontaneous drop of strain energy (Figure 10). Strain energy is the energy stored in the system, and as work is done to the system, typically the strain energy will be increased. However, when buckling happens, the system cannot support the stress added and energy is simultaneously released as heat which induces a sudden decrease in strain energy. Table 1 and Figure 10 show how the total strain energy changes with displacements added for the $2 \times 2 \times 2 \mathrm{~nm} 15 \mathrm{PE}$ chain matrix.

Table 1 Total Strain Energy VS Displacement for 2x2x2 nm 15 PE chain matrix

\begin{tabular}{|l|l|l|l|l|l|l|l|l|l|l|}
\hline Disp $(\AA)$ & 0 & 0.1 & 0.2 & 0.3 & 0.4 & 0.5 & 0.6 & 0.7 & 0.8 & 0.9 \\
\hline Energy & 7182 & 7190 & 7203 & 7219 & 7238 & 7260 & 7284 & 7310 & 7337 & 7367 \\
$(\mathrm{kcal} / \mathrm{mol})$ & & & & & & & & & & \\
\hline Disp $(\AA)$ & 1.0 & 1.1 & 1.2 & 1.3 & 1.4 & 1.5 & 1.6 & 1.7 & 1.8 & 1.9 \\
\hline $\begin{array}{l}\text { Energy } \\
(\mathrm{kcal} / \mathrm{mol})\end{array}$ & 7398 & 7430 & 7464 & 7500 & 7537 & 7575 & 7615 & 7657 & 7700 & 7676 \\
\hline
\end{tabular}




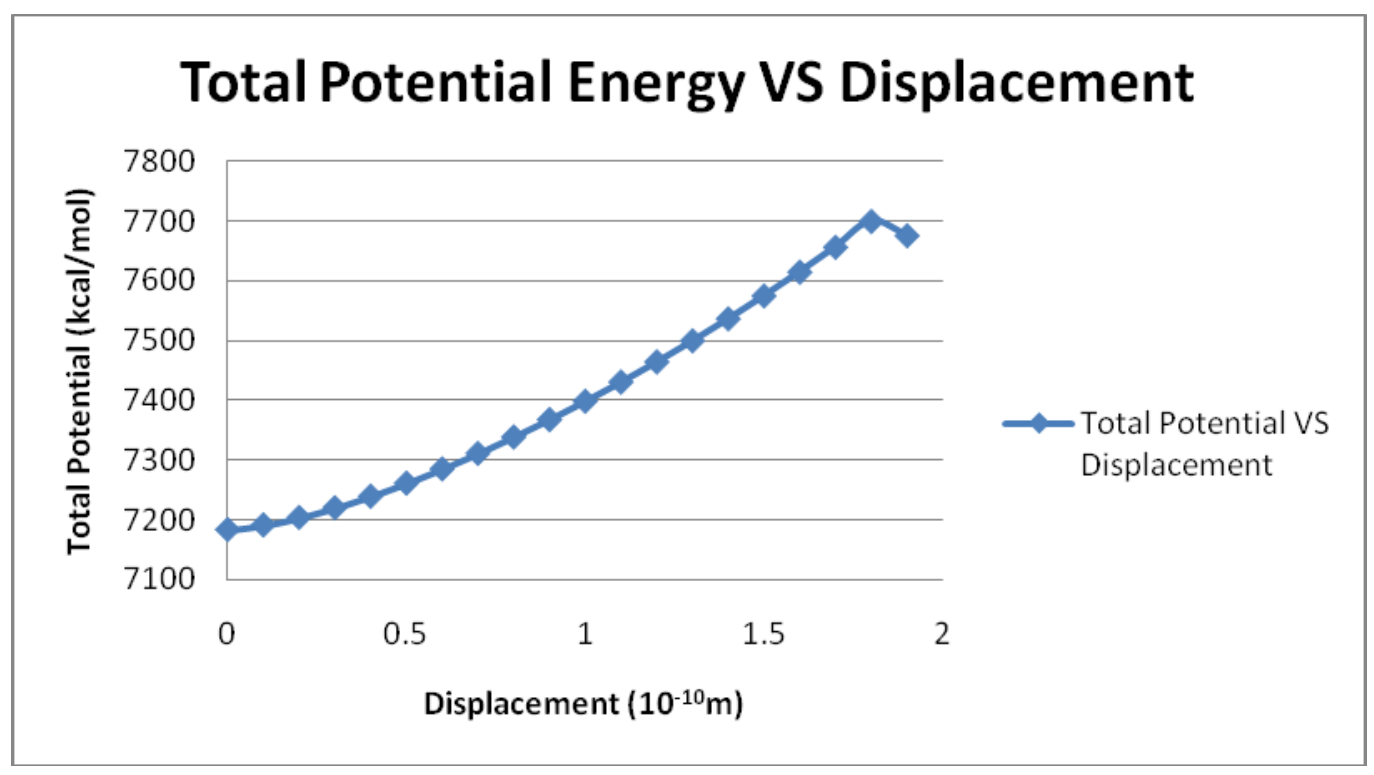

Figure 10 Total potential energy VS displacement for 2x2x2 nm 15 PE chain matrix

3.4.2 The relations between the number of PE chains and the buckling strain After calculating the buckling strains of each individual matrix containing different numbers of PE chains, a comparison of these strains is essential since it will reveal how the buckling strain changes as the number of PE chains changes. Table 2 (a) and (b) are lists of buckling strains of $2 \times 2 \times 2 \mathrm{~nm}$ and $2.5 \times 2.5 \times 2 \mathrm{~nm}$ matrices with ascending numbers of PE chains.

Table 2 (a) Buckling parameters for $2 \times 2 \times 2 \mathrm{~nm}$ matrix

\begin{tabular}{|l|l|l|l|}
\hline $\begin{array}{l}\text { Number of PE } \\
\text { chains }\end{array}$ & Buckling length $(\AA)$ & Buckling Strain & Relative Density \\
\hline 0 (pristine CNT) & 2.3 & $13.14 \%$ & N/A \\
\hline 12 & 2.1 & $12.00 \%$ & 4.210 \\
\hline
\end{tabular}




\begin{tabular}{|l|l|l|l|}
\hline 13 & 2.1 & $12.00 \%$ & 4.561 \\
\hline 14 & 1.9 & $10.86 \%$ & 4.912 \\
\hline 15 & 1.9 & $10.86 \%$ & 5.263 \\
\hline 16 & 1.7 & $9.71 \%$ & 5.614 \\
\hline 17 & 1.6 & $9.14 \%$ & 5.965 \\
\hline 18 & 1.3 & $7.43 \%$ & 6.316 \\
\hline
\end{tabular}

Table 2 (b) Buckling parameters for $2.5 \times 2.5 \times 2 \mathrm{~nm}$ matrix

\begin{tabular}{|l|l|l|l|}
\hline $\begin{array}{l}\text { Number of PE } \\
\text { chains }\end{array}$ & $\begin{array}{l}\text { Length of buckling } \\
(\AA)\end{array}$ & Buckling strain & Relative density \\
\hline 0 (pristine CNT) & 2.3 & $13.14 \%$ & N/A \\
\hline 22 & 2.3 & $13.14 \%$ & 4.207 \\
\hline 23 & 2.2 & $12.57 \%$ & 4.398 \\
\hline 24 & 2.1 & $12.00 \%$ & 4.589 \\
\hline 25 & 2.1 & $12.00 \%$ & 4.780 \\
\hline 26 & 1.9 & $10.86 \%$ & 4.971 \\
\hline 27 & 2.0 & $11.43 \%$ & 5.163 \\
\hline 28 & 1.9 & $10.86 \%$ & 5.354 \\
\hline 29 & 1.5 & $8.57 \%$ & 5.545 \\
\hline
\end{tabular}

The numbers in first column are the PE chain numbers in the matrix where the second and third columns show the length of buckling and buckling strain of the respective 
matrices. The results show that as the number of the PE chains increases, the buckling strains of the matrices will decrease. However, to quantitatively state the relations between buckling strains and PE chain numbers, a new unit called "relative density", defined as the PE chain numbers divided by the area that the PE chains occupy, appears in the last column of Table 2 (a) and (b).

For the $2 \times 2 \times 2 \mathrm{~nm}$ matrix, the area that PE chains occupy is approximately $2.8501 \mathrm{~nm}^{2}$ which is the area of the matrix minus the area of the SWCNT; similarly for $2.5 \times 2.5 \times 2$ matrix, the area the PE chains occupy is about $5.2299 \mathrm{~nm}^{2}$. The results in Table 2 (a) and (b) show that with similar relative density, the buckling strain would be the same. Both 15 PE chain matrix in table 2 (a) and 24 PE chain matrix in table 2 (b) have similar relative density around 4.5 to 4.6 , and they own the same buckling strain which is $12.00 \%$, and within the range of 4.9 to 5.2 for relative density, the buckling strain was about $10.86 \%$ for both matrices. As we only take one standard area of the CNT in each of $2 \times 2 \times 2 \mathrm{~nm}$ matrix and $2.5 \times 2.5 \times 2 \mathrm{~nm}$ matrix and make an assumption that despite the number of PE chains, each matrix has the same CNT area. In the real case, the areas of the CNT in each PE chain matrix are slightly different. However, the influence of the area difference is negligible.

Once the relations between the PE chain numbers and buckling strains have been found, a rational explanation has to be provided. The most acceptable reason is that the vdW interaction between PE chains and CNT affected the buckling strain of the matrix.

One proper prediction is that the vdW interaction between the PE chains and CNT is compressive and the vdW force acted on the CNT body induced an early buckle of the PE-CNT matrix. Therefore the more PE chains added to the matrix, the larger the 
compressive vdW force applied on CNT, which definitely indicates the buckling strain decreases with the number of PE chains increases. However, to verify our prediction, quantitative vdW forces calculation for different PE chain matrices must be done. Contents in chapter 4 have the comparison of vdW forces for different PE chain matrices.

\subsubsection{Factors that affect the results}

Although we already found a reasonable explanation of our results, the applicability of the results has to be discussed. Many factors may affect our results, which include initial position of PE chains and CNT, the range of PE chain numbers, the accuracy of our simulation method and external factors.

The initial position of PE chains and CNT is the first factor that may affect our results. This is because the different initial position of the PE chains will form different matrix shape. The different displacements between the PE chains and the CNT will form different vdW force. The vdW force is one main factor that relates to the buckling strain. Therefore, the initial position would more or less influence the buckling strain. In our model, we have to put the PE in a "regular" initial position so that the buckling strain does not look strange. Figure 11 shows that the shapes of the matrices with same PE chain numbers but different initial positions after minimizing are quite different. To avoid the initial position effect, we always use the models with same initial positions and add or subtract the PE chains on that model. 


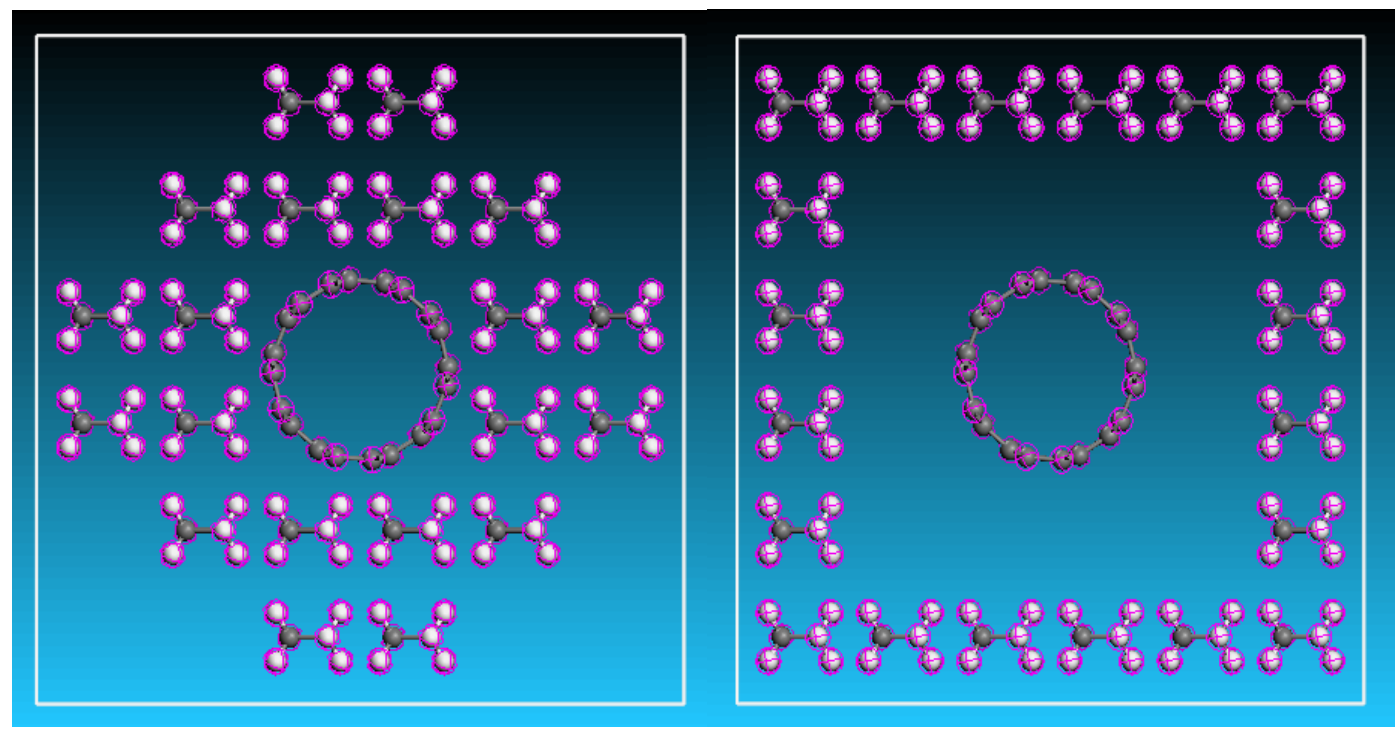

(a)

(b)

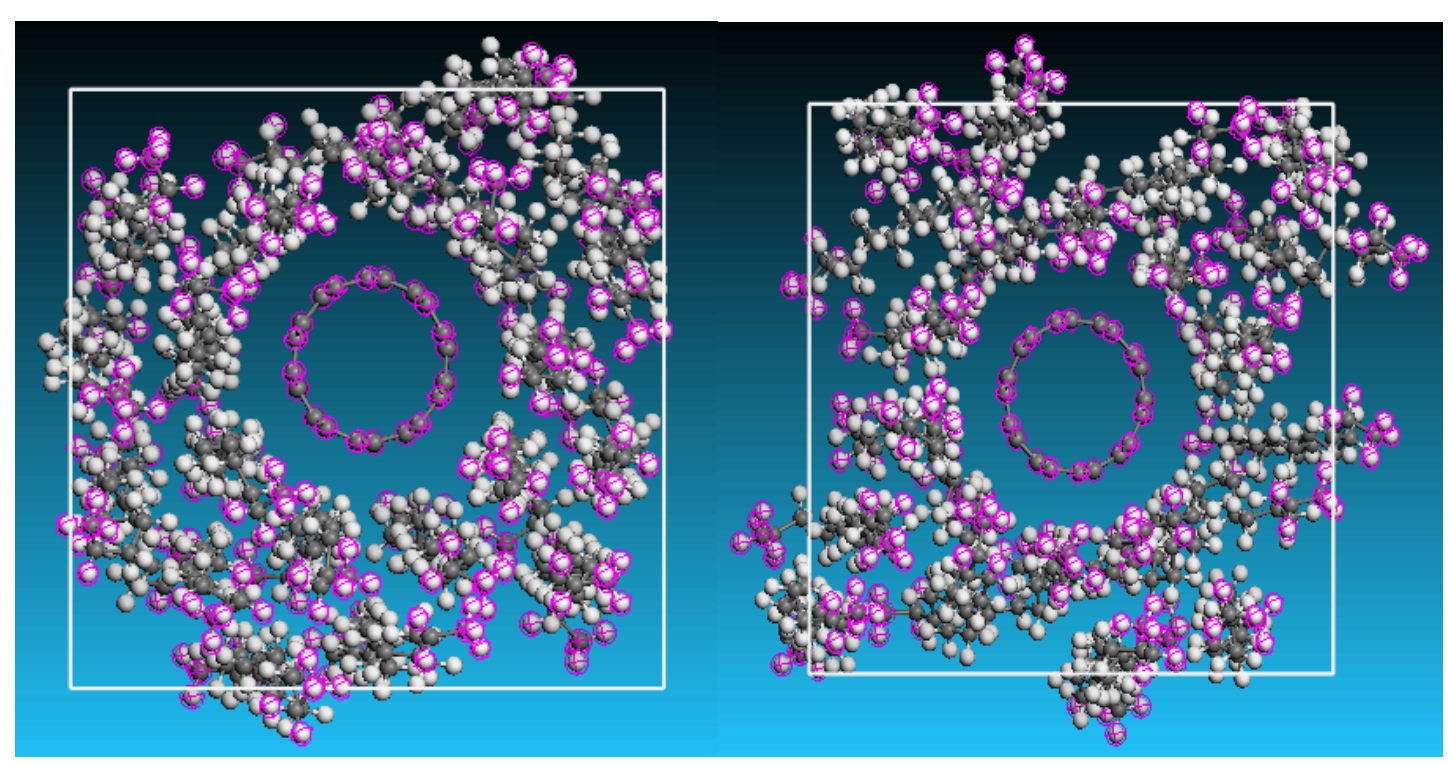

(c)

(d)

Figure 11 (a) One type of initial position for $20 \mathrm{PE}$ Chain in $2.5 \times 2.5 \times 2$ matrix before and (c) after minimizing (b) another type of initial position for 20 PE Chain in $2.5 \times 2.5 \times 2$ matrix before and (d) after minimizing 
The second factor that may affect our results is the PE chain number range. Too many PE chains will make the matrix too crowded and in that case, as the compressive vdW interaction on the CNT is extraordinarily big, the buckling behaviour is more complicated and sometimes very hard to be observed. Moreover, the shape of the CNT will have significant change, i.e. from a circular tube to an ellipse tube. Figure 12 has detailed explanation on how the shape of CNT changes with a higher number of PE chain matrix.

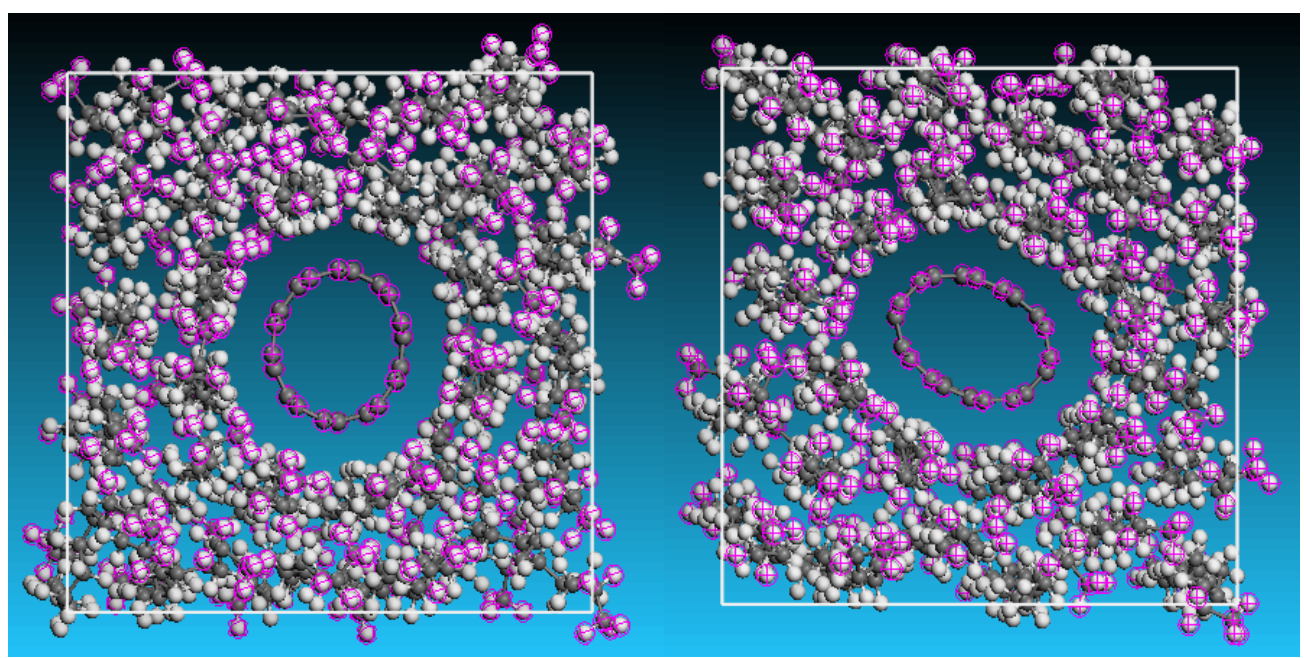

(a)

(b)

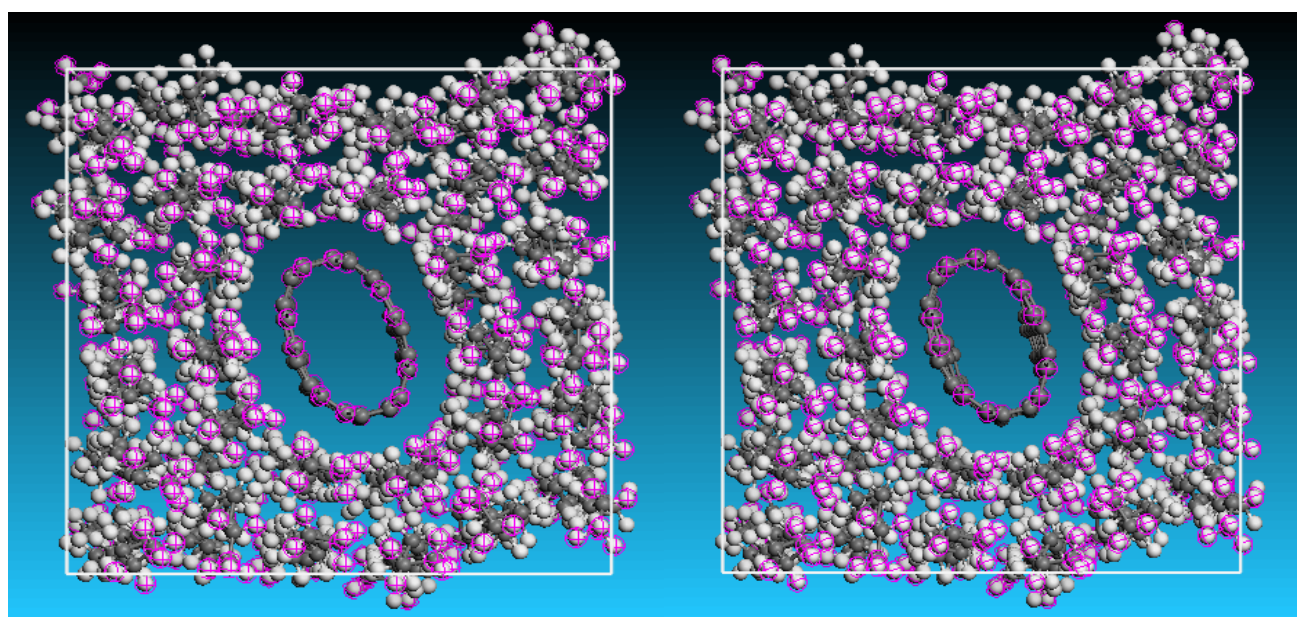

(c)

(d) 
Figure 12 For the $2.5 \times 2.5 \times 2 \mathrm{~nm}$ matrix (a) $28 \mathrm{PE}$ chain matrix and (b) $29 \mathrm{PE}$ chain matrix shape just after minimizing (c) $30 \mathrm{PE}$ chain matrix under $1.1 \AA$ displacement and (d) $12 \AA$ displacement

From Figure 12 (a) and (b), it is found that only one more PE chain is added, the shape of the CNT greatly changed from a circular tube to an ellipse tube. Moreover, from (c) and (d), we found that the buckling behaviour looks very different from the "non-crowed" matrix buckling. This is because the crowded shape induced significantly big compression to the CNT but there is even no space to buckle. However, in this case, the buckling behaviour becomes more complicated and this is the reason that we do not consider the buckling behaviour of a high number of PE chain matrix. The opposite case is the matrix with low number of PE chains. Figure 13 (a) and (b) make a comparison on the shape of $10 \mathrm{PE}$ chain and $12 \mathrm{PE}$ chain in $2 \times 2 \times 2 \mathrm{~nm}$ matrix just after minimizing. From Figure 13 (a), it is obviously seen that there is big "hollow" space in the matrix while Figure 13 (b) had nearly no spare space.

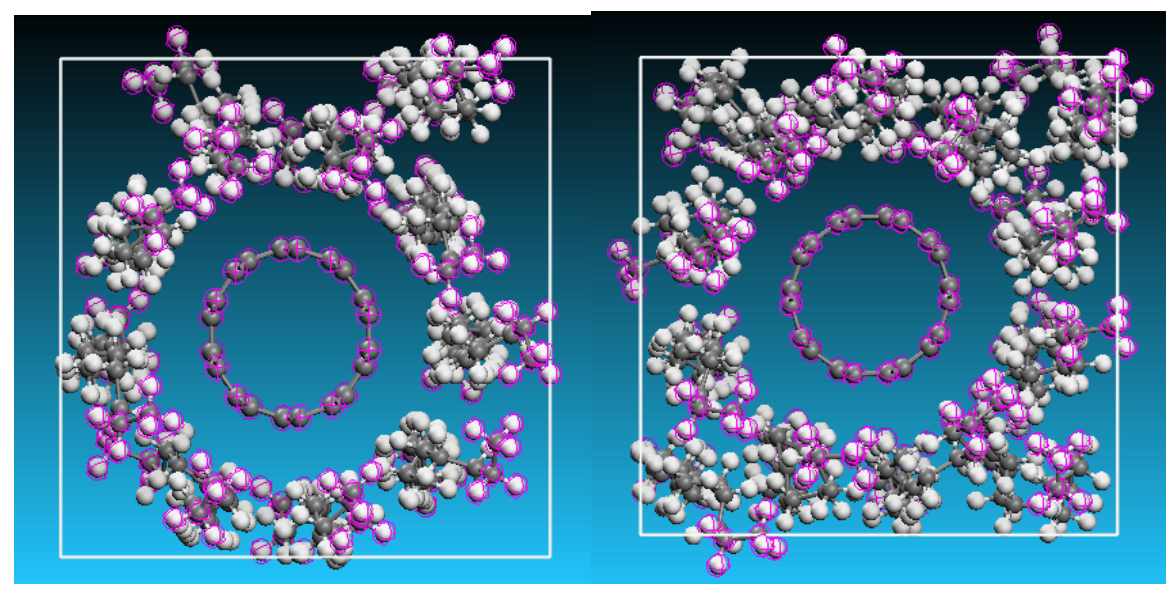

(a)

(b) 
Figure 13 A comparison of the $2 \times 2 \times 2 \mathrm{~nm}$ PE-CNT matrix with (a) 10 chains and (b) 12 chains after minimizing

As there is much spare space in matrix with less PE chains, the variety of different PECNT matrices shapes due to the different initial positions set up of the PE chains will be the main reason of obtaining different buckling strains. This is because the PE-CNT matrices with the same number of PE chains but different shapes will have different vdW interactions between the PE chains and the CNT. Therefore, the buckling behaviours of lower number of the PE chain matrices are not within our consideration. The PE chain numbers we selected in $2 \times 2 \times 2 \mathrm{~nm}$ matrix is from 12 to 18 and in $2.5 \times 2.5 \times 2 \mathrm{~nm}$ matrix is from 22 to 29 .

The third factor which may affect our results is the accuracy of our simulation method. As the method we used to find the buckling strain was by adding specific displacement and minimizing, we could only estimate an approximate buckling strain. For example, the $15 \mathrm{PE}$ chain $2 \times 2 \times 2 \mathrm{~nm}$ matrix owns a buckling length of $1.9 \AA$, which indicated that by adding $1.8 \AA$ the matrix will not buckle but by adding $0.1 \AA$ more it buckles. It does not really mean that just at $1.9 \AA$, it buckled. However, the buckling length must be within the range of $1.8 \AA$ to $1.9 \AA$. Theoretically, we are able to find the most appropriate buckling length by trial and error method. In the real situation, as the calculation time is long, we can only find the buckling length within the error of $0.1 \AA$.

Last, the model we set up can be considered as a general model by employing MM method, which compared to a specific experimental model, neglected a lot of external 
factors such as impurity of the PE matrix, temperature and humidity of synthesis process, etc.

Although most of our data are strong enough to indicate that the buckling strain decreases with the PE chain number increases, one exceptional case in the table 2 (b) were generated on $27 \mathrm{PE}$ chain matrix. The buckling strain here in this case is larger than the matrix with 26 PE chains. The proposed reason could be the software exception when it is employing the COMPASS force field. 


\section{Chapter 4}

\section{VDW force between PE chains and the CNT}

\subsection{General information}

In chapter 3 , it is predicted that the vdW forces between the PE chains and the CNT cause early buckle of the matrix. To verify our prediction, vdW forces in different PE chain matrices have to be obtained. MM method is employed and specific simulation techniques are needed to find the vdW forces between the PE chains and the CNT. Detailed explanations are provided in part 4.3 and relative results and discussions are in part 4.4 .

\subsection{VDW force and Lennard-Jones potential}

In physical chemistry, the vdW force (or vdW interaction), named after Dutch scientist Johannes Diderik van der Waals, is the sum of the attractive or repulsive forces between 
molecules (or between parts of the same molecule) other than those due to covalent bonds or to the electrostatic interaction of ions with one another or with neutral molecules. [52]

The Lennard-Jones potential is often used as an approximate model for the isotropic part of a total (repulsion plus attraction) $\mathrm{vdW}$ force as a function of distance.

$\mathrm{vdW}$ forces are responsible for certain cases of pressure broadening (vdW broadening) of spectral lines and the formation of vdW molecules. The London-vdW forces are related to the Casimir effect for dielectric media, the former being the microscopic description of the latter bulk property. The first detailed calculations of this were done in 1955 by E. M. Lifshitz. [53]

The Lennard-Jones potential (also referred to as the L-J potential, 6-12 potential, or 12-6 potential) is a mathematically simple model that approximates the interaction between a pair of neutral atoms or molecules. A form of the potential was first proposed in 1924 by John Lennard-Jones. [54] The most common expressions of the L-J potential are

$$
\begin{aligned}
V_{L S} & =4 c\left\{\left(\frac{\sigma}{r}\right)^{12}-\left(\frac{\sigma}{r}\right)^{6}\right\} \\
& =e\left\{\left(\frac{P_{m}}{r}\right)^{12}-2\left(\frac{P_{m}}{r}\right)^{6}\right\}
\end{aligned}
$$

where $\varepsilon$ is the depth of the potential well, $\sigma$ is the finite distance at which the interparticle potential is zero, $r$ is the distance between the particles, and $r_{m}$ is the distance at which the potential reaches its minimum. At $r_{\mathrm{m}}$, the potential function has the value $-\varepsilon$. The distances are related as $r_{\mathrm{m}}=2^{1 / 6} \sigma$. These parameters can be fitted to reproduce 
experimental data or accurate quantum chemistry calculations. Due to its computational simplicity, the Lennard-Jones potential is used extensively in computer simulations even though more accurate potentials exist.

\subsection{Simulation}

\subsubsection{Modeling}

As we are calculating the vdW forces for the PE chain matrices, the model is the same as we set up in chapter 3. However, in order to obtain the vdW forces between the PE chains and the CNT, specific simulation method and technique must be employed.

\subsubsection{Simulation technique and process}

From the data obtained in the summary table, the only vdW potential we can directly obtain is the total vdW potential and there is no direct method to find the vdW potential between the PE chains and $\mathrm{CNT}$, i.e. $\mathrm{vdW}_{\mathrm{PE}-\mathrm{CNT}}$. However, if we could find the vdW potential between the atoms on PE chains which was $\mathrm{vdW}_{\mathrm{PE}-\mathrm{PE}}$ and the vdW potential between the atoms on the $\mathrm{CNT}$, $\mathrm{vdW}_{\mathrm{CNT}-\mathrm{CNT}}$, we were able to employ this formula

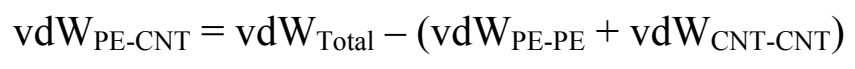

where $\mathrm{vdW}_{\text {Total }}$ is the vdW potential between every atom within the system which was the summation of vdW $\mathrm{vE}_{\mathrm{PE}-\mathrm{CNT}}, \mathrm{vdW}_{\mathrm{PE}-\mathrm{PE}}$ and $\mathrm{vdW}_{\mathrm{CNT}-\mathrm{CNT}}$. However, to get the vdW potential would not be our final approach. We needed to calculate the vdW force using the vdW 
potential. As we know, $\Delta E=F \Delta x$, where $\Delta E$ here is the difference between the two $\mathrm{vdW}$ potentials with adjacent displacements, $F$ is the vdW force acting on the CNT between two adjacent displacements and $\Delta x$ is the change of displacement between CNT and PE chains. In the real case it is very hard to take the accurate data for $\Delta x$, and as an assumption, we considered the shape of the PE chains remain the same which means that $\Delta x$ is only related to the change of CNT shape. This assumption could very much facilitate our calculation.

For the 3 parameters on the right hand side of equation $4.2, \mathrm{vdW}_{\text {Total }}$ can be obtained directly from the energy summary table and $\mathrm{vdW}_{\mathrm{PE}-\mathrm{PE}}$ and $\mathrm{vdW}_{\mathrm{CNT}-\mathrm{CNT}}$ can be found by a very tricky method. First, collect a matrix under specific displacement after minimizing, and then delete the CNT portion and minimizing the rest part. The "Maximum Iterations" should be set as 0 steps, i.e. the final $\mathrm{vdW}$ potential equals the initial vdW potential. The $v d W$ potential generated from the summary table is $\mathrm{vdW}_{\mathrm{PE}-\mathrm{PE}}$. This is up to now the best way to figure out $\mathrm{vdW}$ PE-PE. For $\mathrm{vdW}_{\mathrm{CNT}-\mathrm{CNT}}$ the same method could be applied but the only difference is the deleted portion should be the PE chains other than the CNT. Figure 14 shows the matrices shapes before and after deleting the CNT.

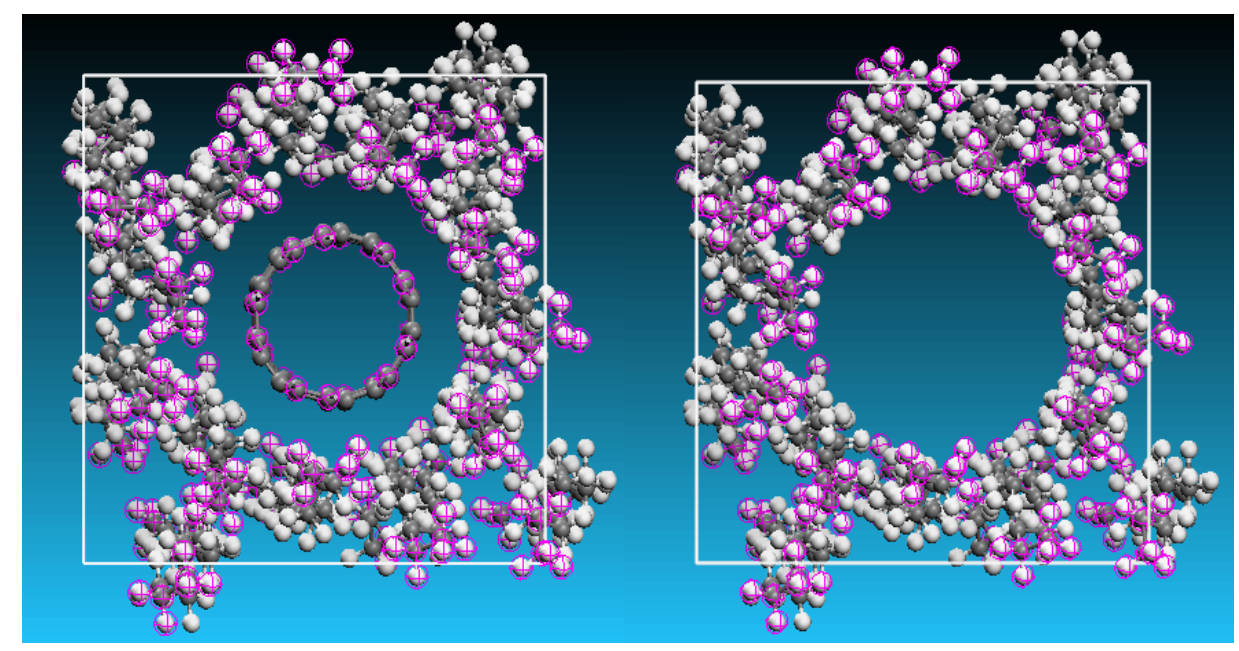


Figure 14 (a) $15 \mathrm{PE}$ chain $2 \times 2 \times 2$ nm matrix under $1.6 \AA$ displacement before (b) and after deleting CNT

\subsection{Results and discussions}

By applying Eqn. (4.2), we obtain PE-CNT vdW potential and the difference of PE-CNT $\mathrm{vdW}$ potential $\Delta E$ between two adjacent displacements. The results of vdW potentials for $15 \mathrm{PE}$ chain $2 \times 2 \times 2 \mathrm{~nm}$ matrix are shown in Table 3 and the Figure 15.

Table 3 The vdW potential for total, PE-PE, CNT-CNT, PE-CNT and the difference for PE-CNT for $15 \mathrm{PE}$ chain $2 \times 2 \times 2 \mathrm{~nm}$ matrix

\begin{tabular}{|c|c|c|c|c|c|}
\hline $\begin{array}{l}\text { Displacement } \\
\left(10^{-10} \mathrm{~m}\right)\end{array}$ & $\begin{array}{l}\text { Total } \\
\text { VDW } \\
(\mathrm{kcal} / \mathrm{mol})\end{array}$ & $\begin{array}{l}\text { PE VDW } \\
(\mathrm{kcal} / \mathrm{mol})\end{array}$ & $\begin{array}{l}\text { CNT } \\
\text { VDW } \\
(\mathrm{kcal} / \mathrm{mol})\end{array}$ & $\begin{array}{l}\text { PE-CNT } \\
\text { VDW } \\
(\mathrm{kcal} / \mathrm{mol})\end{array}$ & Difference \\
\hline 0 & -234.596034 & -232.74 & 147.5592 & -149.415309 & 0.06261 \\
\hline 0.1 & -236.982468 & -232.559 & 144.9297 & -149.352699 & 0.065639 \\
\hline 0.2 & -237.737354 & -232.383 & 143.9328 & -149.28706 & 0.063935 \\
\hline 0.3 & -237.10927 & -232.207 & 144.3207 & -149.223125 & 0.062629 \\
\hline 0.4 & -235.295024 & -232.029 & 145.8948 & -149.160496 & 0.060921 \\
\hline 0.5 & -232.45268 & -231.852 & 148.4985 & -149.099575 & 0.05841 \\
\hline 0.6 & -228.702941 & -231.673 & 152.0117 & -149.041165 & 0.056221 \\
\hline 0.7 & -224.136535 & -231.495 & 156.3437 & -148.984944 & 0.052782 \\
\hline
\end{tabular}




\begin{tabular}{|r|r|r|r|r|r|}
\hline 0.8 & -218.821311 & -231.317 & 161.4276 & -148.932162 & 0.04848 \\
\hline 0.9 & -212.80739 & -231.139 & 167.2151 & -148.883682 & 0.044428 \\
\hline 1 & -206.127481 & -230.962 & 173.6737 & -148.839254 & 0.040081 \\
\hline 1.1 & -198.803183 & -230.786 & 180.7822 & -148.799173 & 0.034117 \\
\hline 1.2 & -190.849187 & -230.613 & 188.5287 & -148.765056 & 0.025775 \\
\hline 1.3 & -182.27155 & -230.44 & 196.9078 & -148.739281 & 0.015594 \\
\hline 1.4 & -173.074333 & -230.27 & 205.9195 & -148.723687 & 0.000615 \\
\hline 1.5 & -163.255643 & -230.101 & 215.5681 & -148.723072 & -0.019177 \\
\hline 1.6 & -152.8111 & -229.93 & 225.8613 & -148.742249 & -0.045328 \\
\hline 1.7 & -141.728071 & -229.754 & 236.8135 & -148.787577 & -0.097028 \\
\hline 1.8 & -130.006169 & -229.568 & 248.4468 & -148.884605 & 6.843512 \\
\hline 1.9 & -128.043042 & -233.614 & 247.6124 & -142.041093 & \\
\hline
\end{tabular}

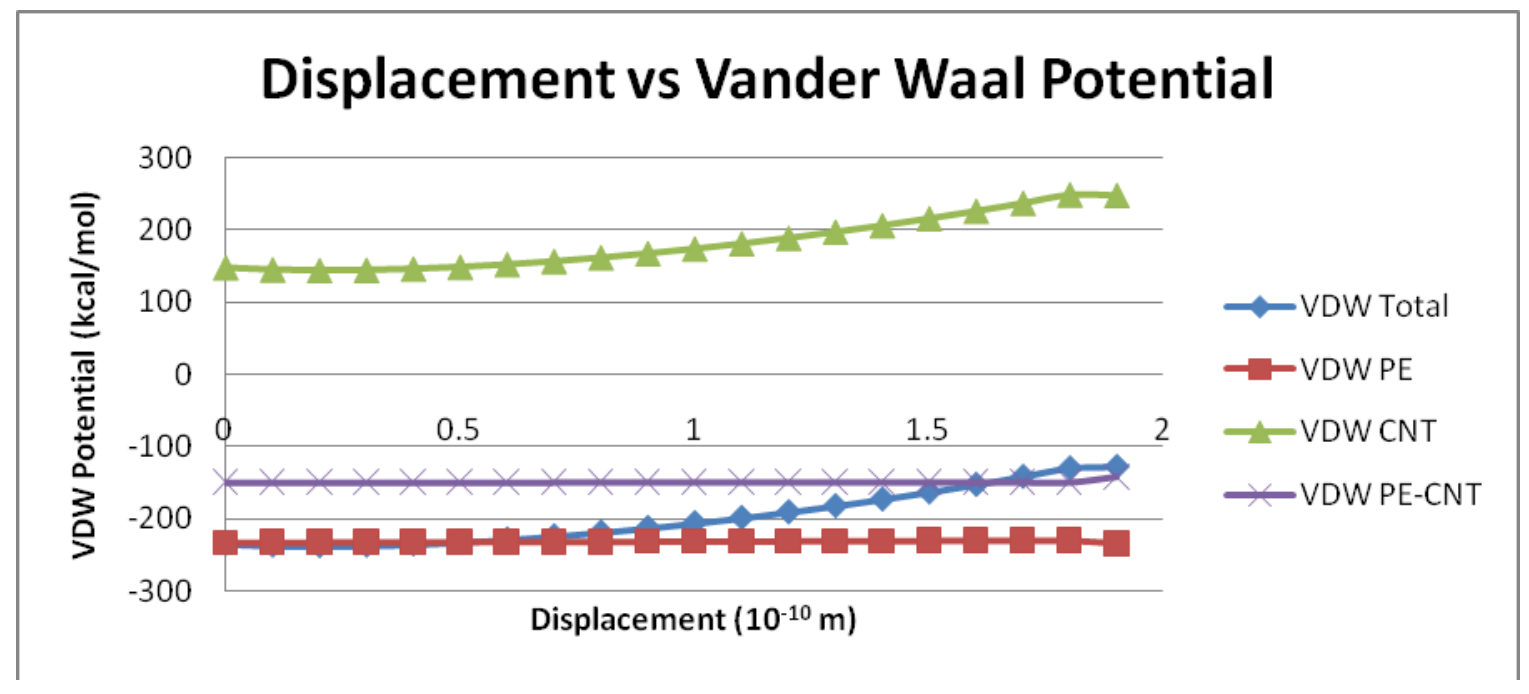

Figure 15 The vdW potential for total, PE-PE, CNT-CNT, PE-CNT and the difference for PE-CNT for $15 \mathrm{PE}$ chain $2 \times 2 \times 2 \mathrm{~nm}$ matrix in curve 
From Figure 15 , it is clear to see that at $1.9 \AA$, the vdW potentials for total, PE-PE, CNTCNT, PE-CNT significantly changed, which can also indicate the moment when buckling happens. Our purpose is to find the vdW force before buckling, and we only take the vdW potentials and differences at $0.3 \AA, 0.2 \AA$ and $0.1 \AA$ before buckling. Table 4 is the data collected from the summary table.

Table 4 Summary table parameters collected for $2 \times 2 \times 2 \mathrm{~nm}$ matrices

\begin{tabular}{|c|c|c|c|c|c|}
\hline 12Chain & $\mathrm{Kcal} / \mathrm{mol}$ & $\mathrm{Kcal} / \mathrm{mol}$ & $\mathrm{Kcal} / \mathrm{mol}$ & $\mathrm{Kcal} / \mathrm{mol}$ & $\mathrm{Kcal} / \mathrm{mol}$ \\
\hline \multicolumn{6}{|l|}{ Displacement } \\
\hline$(\AA)$ & Total & $\mathrm{PE}$ & CNT & PECNT & Difference \\
\hline 1.7 & -93.216 & -188.744 & 234.6735 & -139.146 & -0.096831 \\
\hline 1.8 & -81.8013 & -188.839 & 246.2808 & -139.243 & -0.110568 \\
\hline 1.9 & -69.7139 & -188.929 & 258.5683 & -139.353 & -0.137591 \\
\hline 2 & -56.9339 & -189.002 & 271.5592 & -139.491 & \\
\hline \multicolumn{6}{|l|}{ 13Chain } \\
\hline \multicolumn{6}{|l|}{ Displacement } \\
\hline$(\AA)$ & Total & $\mathrm{PE}$ & CNT & PECNT & Difference \\
\hline 1.7 & -140.068 & -233.943 & 234.8082 & -140.932 & -0.161291 \\
\hline 1.8 & -128.542 & -233.862 & 246.4138 & -141.094 & -0.175103 \\
\hline 1.9 & -116.322 & -233.751 & 258.6982 & -141.269 & -0.183034 \\
\hline 2 & -103.381 & -233.609 & 271.6803 & -141.452 & \\
\hline 14Chain & & & & & \\
\hline
\end{tabular}




\begin{tabular}{|c|c|c|c|c|c|}
\hline $\begin{array}{l}\text { Displacement } \\
(\AA)\end{array}$ & Total & $\mathrm{PE}$ & CNT & PECNT & Difference \\
\hline 1.5 & -179.015 & -237.897 & 214.3096 & -155.427 & 0.064413 \\
\hline 1.6 & -168.539 & -237.747 & 224.5705 & -155.363 & 0.079792 \\
\hline 1.7 & -157.31 & -237.5 & 235.4726 & -155.283 & 0.096691 \\
\hline 1.8 & -145.292 & -237.133 & 247.0271 & -155.186 & \\
\hline \multicolumn{6}{|l|}{ 15Chain } \\
\hline \multicolumn{6}{|l|}{ Displacement } \\
\hline$(\AA)$ & Total & PE & CNT & PECNT & Difference \\
\hline 1.5 & -163.256 & -230.101 & 215.5681 & -148.723 & -0.019177 \\
\hline 1.6 & -152.811 & -229.93 & 225.8613 & -148.742 & -0.045328 \\
\hline 1.7 & -141.728 & -229.754 & 236.8135 & -148.788 & -0.097028 \\
\hline 1.8 & -130.006 & -229.568 & 248.4468 & -148.885 & \\
\hline \multicolumn{6}{|l|}{ 16Chain } \\
\hline \multicolumn{6}{|l|}{ Displacement } \\
\hline$(\AA)$ & Total & PE & CNT & PECNT & Difference \\
\hline 1.4 & -161.604 & -218.818 & 207.4897 & -150.276 & 0.282985 \\
\hline 1.5 & -150.247 & -217.332 & 217.0782 & -149.993 & -2.488037 \\
\hline 1.6 & -141.941 & -216.681 & 227.2206 & -152.481 & 0.41766 \\
\hline 1.7 & -129.858 & -215.79 & 237.9947 & -152.063 & \\
\hline \multicolumn{6}{|l|}{ 17Chain } \\
\hline $\begin{array}{l}\text { Displacement } \\
(\AA)\end{array}$ & Total & PE & CNT & PECNT & Difference \\
\hline 1.3 & -78.4566 & -134.661 & 199.7421 & -143.538 & 0.398851 \\
\hline
\end{tabular}




\begin{tabular}{|c|c|c|c|c|c|}
\hline 1.4 & -69.0284 & -134.704 & 208.8148 & -143.139 & 2.3949 \\
\hline 1.5 & -58.5262 & -136.489 & 218.707 & -140.744 & 0.48665 \\
\hline 1.6 & -48.1532 & -136.98 & 229.084 & -140.257 & \\
\hline 18Chain & & & & & \\
\hline $\begin{array}{l}\text { Displacement } \\
(\AA)\end{array}$ & Total & PE & CNT & PECNT & Difference \\
\hline 0.9 & 4.749292 & -30.1393 & 171.2846 & -136.396 & 0.368133 \\
\hline 1 & 12.0096 & -29.6128 & 177.6503 & -136.028 & 0.418488 \\
\hline 1.1 & 20.08549 & -28.976 & 184.6708 & -135.609 & 0.522052 \\
\hline 1.2 & 29.10407 & -28.146 & 192.3374 & -135.087 & \\
\hline
\end{tabular}

From Table 4, it is very clear to see that the vdW potential difference for 12,13 and 15 PE chains are negative, which means that tensile vdW forces apply between PE chains and CNT. It is strange that these results conflict with our prediction stating that occurrence of buckling is due to the compressive forces between the PE chains and CNT. It is because, primarily, our assumption is that the shapes of PE chains do not change with the displacements adding up to the matrices. In fact, this assumption does not apply to matrices with less PE chains. In another word, the shapes of PE chains with less PE chains will change in less PE chains matrices. As a comparison, the relative density is high enough for matrices with 16 or more PE chains. It is because the matrices are too crowded and there is no more space to move; therefore, our assumption applies. In this case, matrices with less than 16 PE chains will not be considered in calculation. 
One exception is at 16 Chain matrix under $1.6 \AA$ displacement and the other is at 17 Chain matrix under $1.5 \AA$ displacement. Both of the two exceptions have similar results, which is the vdW potential significantly changed, either positive or negative. The reason is that the shape of the CNT changed slightly when exceptions occurred but did not buckle. Figure 16 has the detailed comparison.

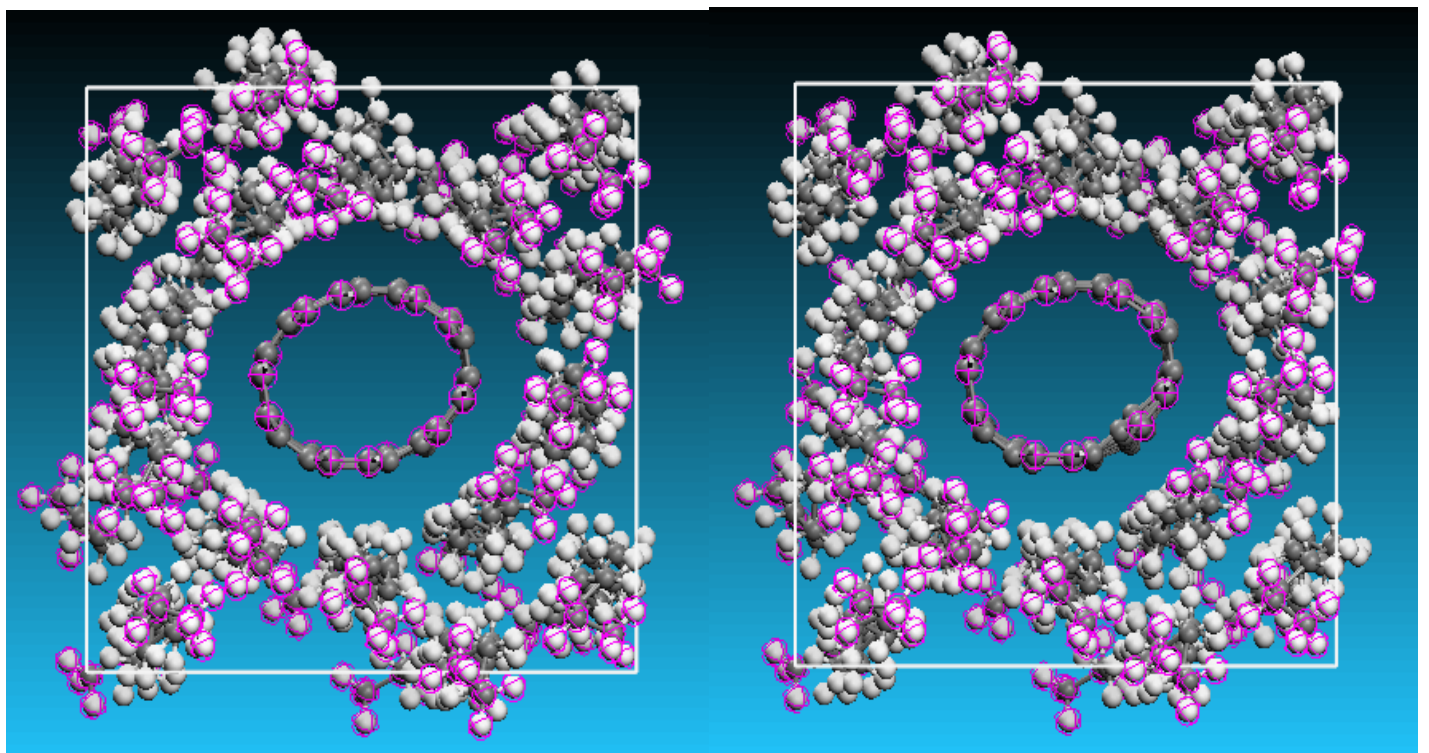

(a)

(b)

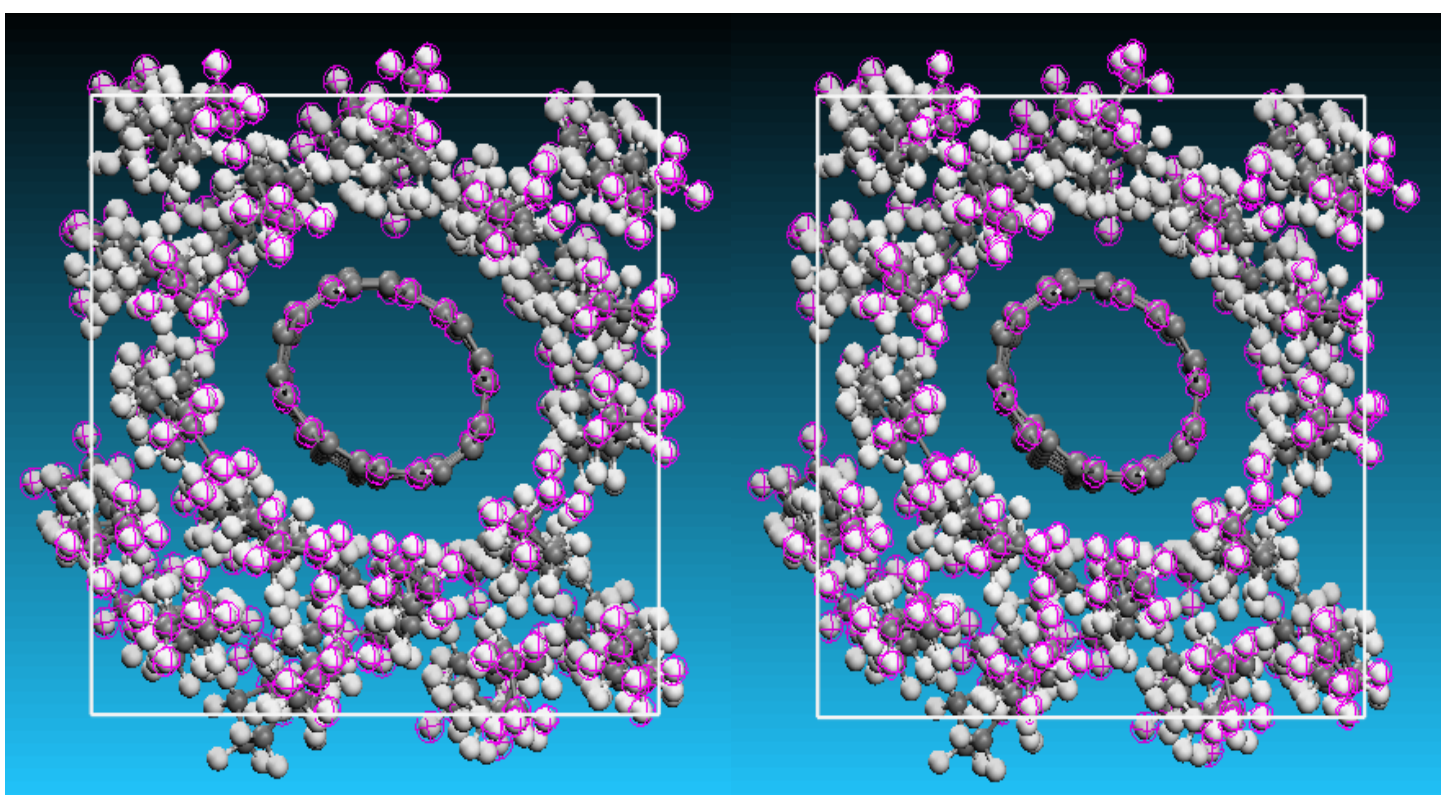


Figure 16 (a) 16 PE Chain 2x2x2 matrix under $1.5 \AA$ (b) and $1.6 \AA$ displacement (c) 17 PE Chain 2x2x2 nm matrix under $1.4 \AA$ and $1.5 \AA$ displacements

From Figure 16 (b) and (d) it is obviously seen that the shapes of the CNT ends have already changed due to compression. In the calculation, these two exceptions will not be collected. The method that we collected the displacement is first to choose two sets of data of two atoms diametrically and found the planer average distance, then divided by 2 to get the average displacement of a single atom. After all, use the vdW potential to divide this displacement and we will get the vdW force. Finally, with the unit conversion, the vdW forces for 16, 17 and $18 \mathrm{PE}$ chain matrices were $2.74496 \mathrm{nN}, 3.41699 \mathrm{nN}$ and $6.95629 \mathrm{nN}$, respectively. The results calculated show that the compressive vdW forces between PE Chains and CNT are becoming bigger and bigger as the chain number increases, which can directly prove that the compressive vdW force is the major factor that influences the buckling strain. Moreover, the results point out that as the PE chain number increases, the compressive vdW forces between the PE chains and CNT will increase, and the buckling strains will decrease. 


\section{Chapter 5}

\section{Vibrations of PE-CNT matrix}

\subsection{Introduction}

Vibration is a very important mechanical behaviour for CNTs and their composites. In this chapter, the relations between the resonance frequencies and PE chain numbers will be obtained and then explained by the vdW interactions between the PE chains and CNT by applying a continuum model.

\subsection{Continuum vibration model}

It is important to have accurate theoretical models for the natural frequencies and mode shapes of CNTs for several reasons. For example, if the nanotubes are to be used as nanomechanical resonators, the oscillation frequency is a key property of the resonator. In addition, the effective elastic modulus of a nanotube may be indirectly determined 
from its measured natural frequencies or mode shapes if a sufficiently accurate theoretical model is used. A continuum approach using the well known frequency equation for a beam has been employed by several investigators to estimate the theoretical fundamental flexural resonance frequencies of nanotubes [55] and nanowires [56]. For example, according to the well-known Bernoulli-Euler beam theory, the equation describing transverse, or flexural motion of a continuous, homogeneous, isotropic, linear elastic beam which is without rotary inertia or shear effects and whose properties do not vary along its length may be expressed as

$$
E I \frac{\partial^{4} w}{\partial x^{4}}+\rho A \frac{\partial^{2} w}{\partial t^{2}}=0
$$

where $\mathrm{E}$ is the effective Young's modulus of beam material, I the moment of inertia of beam cross-section about its neutral axis, A the cross-sectional area of beam, $\rho$ the mass density of beam material, $\mathrm{x}$ the distance along beam, $\mathrm{w}(\mathrm{x}, \mathrm{t})$ the transverse displacement of beam and $t$ is the time. The corresponding frequency equation for flexural vibration is

$$
f_{n}=\frac{\left(k_{n} L\right)^{2}}{2 \pi L^{2}}\left(\frac{E I}{\rho A}\right)^{1 / 2}
$$

where $\mathrm{L}$ is the length of beam, $\mathrm{n}$ the mode number, $\mathrm{knL}$ the eigenvalue for $\mathrm{nth}$ mode and $\mathrm{fn}$ is the nth mode frequency, Hz. The eigenvalues depend on the boundary conditions, and the choice of boundary conditions is obviously very important for an accurate simulation. For example, the first three eigenvalues for a fixed-free cantilever beam are $k_{1} L=1.875, k_{2} L=4.694, k_{3} L=7.855$, whereas the corresponding values for a free-free beam are $k_{1} L=4.73, k_{2} L=7.853, k_{3} L=10.995$. Eigenvalues and the corresponding 
mode shape functions have been tabulated by Blevins [57] for the first few modes and the most commonly encountered boundary conditions.

Yoon et al. [58] developed a double Timoshenko beam model to characterize the motions of the inner and outer tubes in a double wall CNT and then used the model to study the effects of rotary inertia and shear deformation on terahertz frequency transverse wave propagation in double wall CNTs. It was concluded that, because of the relative motions between the inner and outer tubes at high frequencies, the Timoshenko beam model is more relevant than the Bernoulli-Euler model for terahertz frequency wave propagation in double wall CNTs. A similar Timoshenko beam model was used to study terahertz frequency vibrations of short CNTs [59].

The resonance frequency of a typical single SWNT vibrating in the free-free mode is easily in the GHz range, and when the nanotube is embedded in a polymer matrix which provides elastic restraint, the frequencies are even greater. For MWNTs embedded in an elastic medium such as a polymer matrix, the effect of the matrix on the resonance frequencies of the MWNT has been estimated by using a beam-on-elastic foundation model [60]. In this model, Eqn. (5.3) modified by adding a distributed elastic reaction force to the last of Eqn. (5.3) which applies to the outer tube in a MWNT, as

$$
p-c_{N-1}\left[w_{N}-w_{N-1}\right]=E I_{N} \frac{\partial^{4} w_{n}}{\partial x^{4}}+\rho A_{N} \frac{\partial^{2} w_{n}}{\partial t^{2}}
$$

where $\mathrm{p}=-\mathrm{Kw}_{\mathrm{N}}$ is a Winkler-type elastic reaction force per unit length and $\mathrm{K}$ is a constant which depends on the material in which the MWNT is embedded. The results of this simulation show that when the elastic medium surrounding the MWNT is very compliant, the vibrational modes consist of both global flexure and noncoaxial intertube 
deformations. However, when the elastic medium is very stiff, almost all of the vibrational modes are essentially of the noncoaxial intertube type. Arash and Ansari [61] assessed the effectiveness of the present nonlocal shell model by MD simulations as a benchmark of good accuracy and the fundamental resonant frequencies from continuum shell model and MD simulations for clamped and cantilever SWCNTs of thickness $\mathrm{h}=0.34 \mathrm{~nm}$ is found in figure 4.

However, using a tight binding MD simulation of the same problem, Dereli and Ozdogan [62] observed the same induced radial vibration at the same frequency, but found it to be sensitive to tensile strain rate. Ab initio calculations of structural, elastic and vibrationalproperties of SWNTs have included the effects of chirality and radius [63]

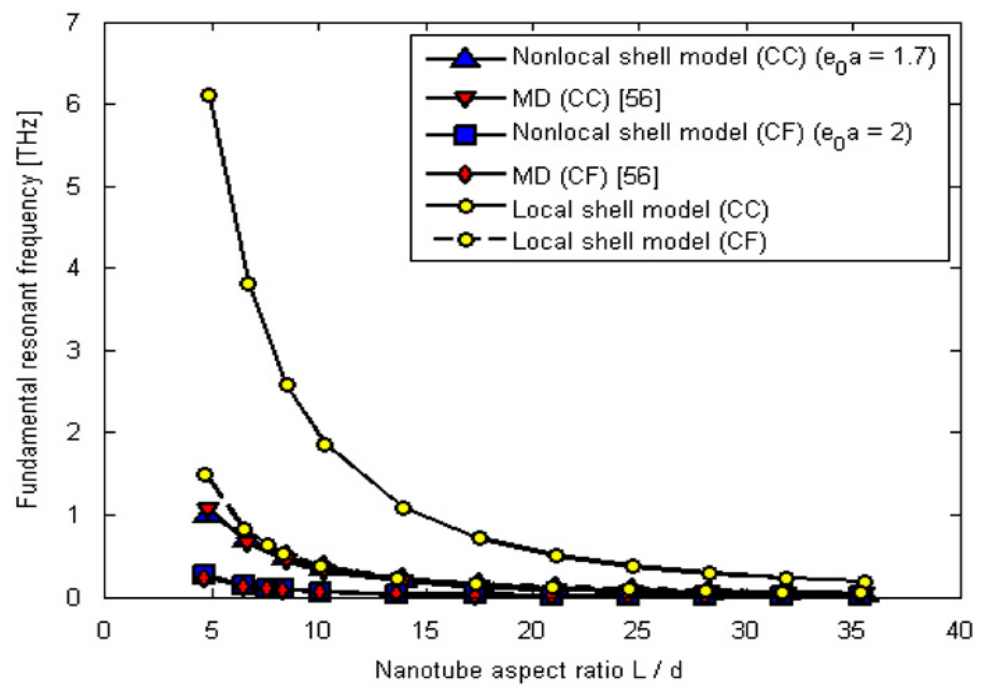

Figure 17 Fundamental resonant frequencies from continuum shell model and MD simulations for clamped and cantilever SWCNTs of thickness $\mathrm{h}=0.34 \mathrm{~nm}$. [61] 


\subsection{Simulation process}

\subsubsection{Modeling}

The model we used is the $2 \times 2 \times 2 \mathrm{~nm}$ matrix which is exactly the same as we set up in the buckling behaviour section. The matrices with SWCNT only, and 1, 2, 4, 6, 8, 12 and 13 PE chains are setup.

\subsubsection{Minimization and vibration analysis}

First, select the compass force field. Second, check the box of "Include Hessian". Third, on the "Job control" tab, select 1 processor instead of 4 in parallel. This is because the software does not support parallel calculation including Hessian. No constraints or displacements are added. Only use the minimizing function and set the maximum iterations to be 300000 to ensure that the atoms find their balanced position. Afterwards, press the "calculation" button under energy tab and make sure that the "Include Hessian" box remains checked. Finally, on the toolbar, press "tools" and click on "Vibrational Analysis", then click on the "calculate" modes. A list of vibration frequencies from low to high will be listed. Double click each frequency, and different mode shapes will display. Figure 19 shows the shape of the matrix including 1 PE chain before and after minimizing. 

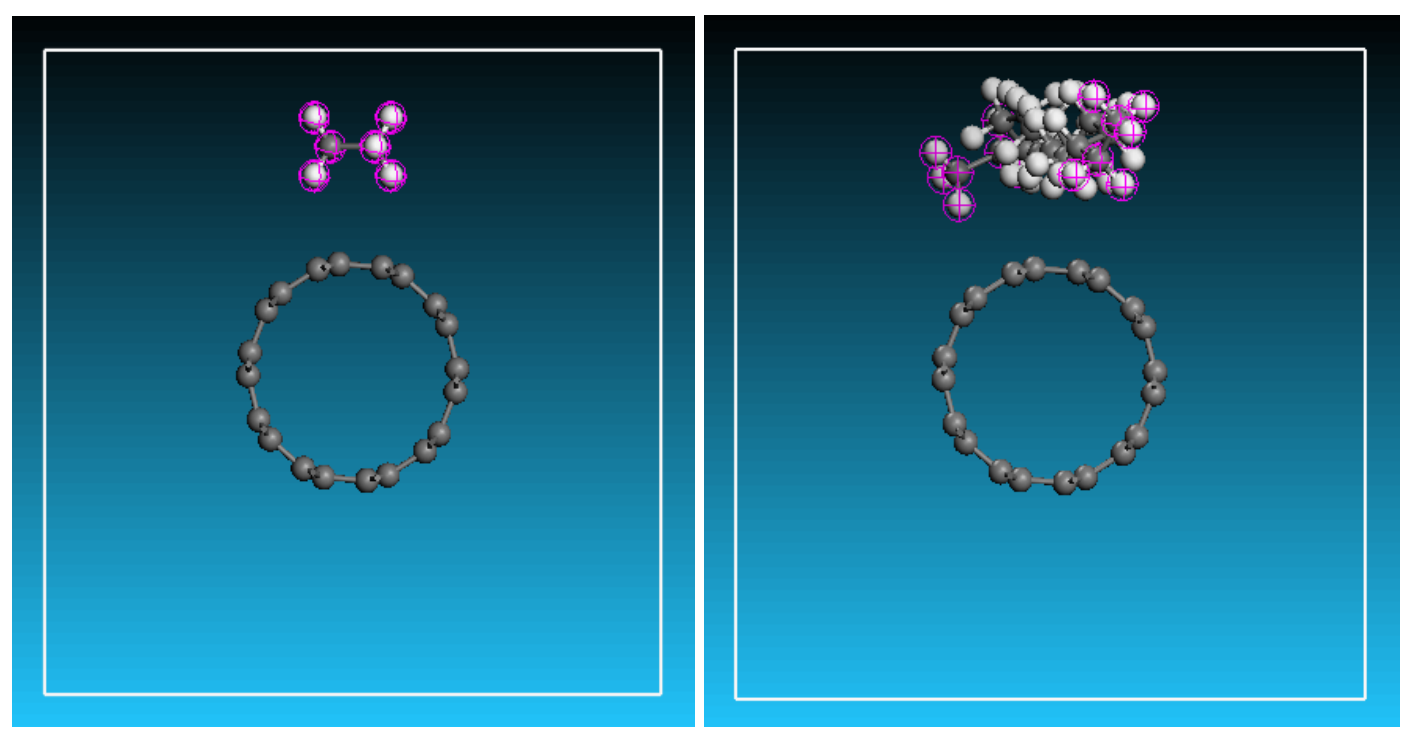

Figure 18 (a) the shape of the matrix including $1 \mathrm{PE}$ chain before (b) and after minimizing

\subsection{Results and discussions}

A list of frequencies will be generated. For example, the matrix with $1 \mathrm{PE}$ chain will generate the range of frequencies from 4.85 up to $2981 \mathrm{~cm}^{-1}$ and totally 644 groups of data. The unit $\mathrm{cm}^{-1}$ is the reciprocal of wave length. In another word, the numbers of wave periods included in the $1 \mathrm{~cm}$ length. To calculate the frequency in $\mathrm{Hz}$, multiplying $3 \times 10^{10}$ is conducted.

Our purpose is to find the fundamental mode of the matrix, i.e. the "breath" type mode. However, as the system can be considered as a huge multi-degree of freedom vibration system with thousands of atoms. Usually, it is very hard to figure out the fundamental mode since there are hundreds of data. However, from the matrix with only SWCNT and no PE chains we found that the breath mode owns the lowest frequency which is 33.36 
$\mathrm{cm}^{-1}$. Therefore, we should try from low to high for every matrix to find the fundamental vibrational modes. Table 5 has the data for the resonance frequencies for matrices containing $0,1,2,4,6,8,12$ and 13 Chains.

Table 5 Resonance frequencies for matrices containing $0,1,2,4,6,8,12$ and 13 chains.

\begin{tabular}{|l|l|}
\hline Number of PE chains & Frequency $\left(\mathrm{cm}^{-1}\right)$ \\
\hline 0 & 33.36 \\
\hline 1 & 29.57 \\
\hline 2 & 24.63 \\
\hline 4 & 24.54 \\
\hline 6 & 22.84 \\
\hline 8 & 22.27 \\
\hline 12 & 21.42 \\
\hline 13 & 20.24 \\
\hline
\end{tabular}

It has been found that with the number of PE chains increases, the fundamental frequency decreases. These results calculated using simulation software coincide very well with the continuum model.

From eqn (5.7) $\omega_{n}=\frac{n \pi}{L} \sqrt{\frac{E I}{\rho A}} \sqrt{\frac{n^{2} \pi^{2}}{L^{2}}-\frac{P}{E I}}$, it is clear to see that the vdW force is inversely proportional to the natural frequency of the PE-CNT matrix. Therefore, as the number of PE chains increases, the resonance frequencies will decrease, which inosculate with the data obtained in Table 5 . 
In fact, we can only calculate the matrices containing up to $13 \mathrm{PE}$ chains. This is due to the calculation ability of the computer. The calculation of matrices with $14 \mathrm{PE}$ chains or more will induce a calculation crush. 


\section{Chapter 6}

\section{Conclusion remarks and future work}

\subsection{Conclusion remarks}

The research on the buckling behaviour of PE-CNTs matrices is significant and a research on how the density of PE chains related to the buckling strain is first discovered. This is not only a significant discovery in research area, but also important in industry manufacturing. For example, by adding a proper amount of CNTs into the PE matrix in the real manufacturing process will be intensively related to our work.

Another significant work is that the vdW forces are calculated between the PE chains and CNT for different number of PE chain matrices. The significance of the job is that it provides quantitative explanation on why the buckling strain decreases with the number of PE chains increases in a PE-CNTs matrix.

The last work that is conducted in the thesis is the analysis of vibration modes for different PE chain matrices. This is the first vibrational modal analysis on PE-CNT matrix and dynamic characteristics of PE-CNT matrix are discovered. 


\subsection{Future work}

Based on the contents of the thesis, it is found that a lot more future work can be done to improve my current work.

\subsubsection{Model improvement}

The model we are taken has high weight percentage of CNT. In real manufacturing situation, the percentage of the CNT in the matrix is usually $5 \%$ up to $40 \%$. The results for lower weight percentage of CNT matrices might be a little different. Although it is found that with the same relative density, the buckling strain is similar, there might be exceptions after we add more PE chains and expand the dimensions of our matrices. However, it may require a lot more time for the computer to calculate the mechanical parameters for larger matrices and more atoms. One way to solve this problem is to purchase better computers with faster CPU calculation since we found that the bottleneck of the calculation is on CPU and the overall usage of the CPU will get to $95 \%-100 \%$ calculation occupation. Another way to solve this is to update the software since our version is old and a new version of Materials Studio may optimize the calculation method which may much more increase the calculation speed.

\subsubsection{Beamlike buckling}

It is clearly seen in the thesis that we only use short CNTs which will induce shell-like buckling. Moreover, for SWCNT, beamlike buckling is another type of buckling which always happen with larger aspect ratio. It is expected that beamlike buckling behaviour for PE-CNTs matrices will have similar results with shell-like buckling behaviour. However, simulations must be done to verify our expectation. One difficulty for the 
simulations of the matrices with larger aspect ratio for CNT is when more atoms are added in, the calculation will be more complicated and much slower. Therefore to improve the work station is very necessary. If use the same computer, the model will have even less PE chains and the weight percentage of the SWCNT will be even larger which means that the model is not that appropriate compared with the real PE-CNTs products manufactured from industries.

\subsubsection{Experimental method}

The experimental method can be done to verify the answers calculated from the simulation software. However, more difficulties will be conquered. First of all, as the real PE-CNT product will not be as perfect as the model we set up, errors may be induced. Second of all, as the differences of buckling strain for different number of PE chains obtained from the simulation result are very small, the buckling differences we got from results we derived from the experiment might not be significant. The third difficulty is that in the experiment, it is very hard to apply accurate displacement to the matrix which is a big barrier to compare the simulation results and the experimental results. 


\section{Bibliography}

[1] Iijima, S., 1991, "Helical Microtubules of Graphitic Carbon," Nature (London), 354, pp. 56-58.

[2] Overney G, Zhong W, Tomanek D. "Structural rigidity and low frequency vibrationalmodes of long carbon tubules". Z Phys D—At Mol Clusters 1993;27(1):93-6

[3] Yu, Min-Feng; Lourie, Oleg; Dyer, Mark J.; Moloni, Katerina; Kelly, Thomas F.; Ruoff, Rodney S. (28 January 2000). "Strength and Breaking Mechanism of Multiwalled Carbon Nanotubes Under Tensile Load". Science 287 (5453): 637-640

[4] Mizutania T, Ohno Y, Kishimoto S., 2008, "Electrical properties of carbon nanotube FETs,” 2008 International Conference on Advanced Semiconductor Devices and Microsystems, 1-8, 2008

[5] J. Hone, M. Whitney, C, Piskoti et al., "Thermal conductivity of single-walled carbon nanotubes", Phys. Rev. B, 59, R2514 (1999)

[6] P. Kim, L. Shi, A. Majumdar et al., "Thermal transport measurements of individual multiwalled nanotubes”, Phys.Rev.Lett., 87, 215502 (2001)

[7] Yakobson, B. I., Brabec, C. J., and Bernholc, J., 1996, "Nanomechanics of Carbon Tubes: Instabilities Beyond Linear Response,” Phys. Rev. Lett., 76, pp. 2511-2514. 
[8] Liew, K. M., Wong, C. H., He, X. Q., Tan, M. J., and Meguid, S. A., 2004, “Nanomechanics of Single and Multiwalled Carbon Nanotubes," Phys. Rev. B, 69, p. 115429.

[9] Iijima, S., Brabec, C., Maiti, A., and Bernholc, J., 1996, "Structural Flexibility of Carbon Nanotubes,” J. Chem. Phys., 104, pp. 2089-2092.

[10] Wang, Q., Liew, K.M., He, X.Q., Xiang, Y., "Local buckling of carbon nanotubes under bending,” Applied Physics Letters 92 (5): 059902

[11] Wang, Q., 2008, "Torsional buckling of double-walled carbon nanotubes," Carbon $46: 1172-1174$

[12] Kilbride BE, Coleman JN, Fraysse J, Fournet P, Cadek M, Drury A, et al. "Experimental observation of scaling laws for alternating current and direct current conductivity in polymer-carbon nanotube composite thin films". J Appl Phys 2002; 92:4024-30.

[13] Biercuk MJ, Llaguno MC, Radosavljevic M, Hyun JK, Johnson AT. "Carbon nanotube composites for thermal management". Appl Phys Lett 2002; 80(15):2767-9.

[14] Wang, Q., "Buckling of carbon nanotubes wrapped by polyethylene molecules," Physics Letters A 2011; 375: 624-627

[15] Thostenson, E.T., Tsu-Wei Chou, "Nanotube buckling in aligned multi-wall carbon nanotube composites," Carbon, 2004; v 42, n 14, 3015-18

[16] Koratkar, NA., Wei, BQ., Ajayan, PM., "Multifunctional structural reinforcement featuring carbon nanotube films," Compos Sci Technol 63(2003) pp. 1525-1531.

[17] Koratkar, NA., Wei, BQ., Ajayan, PM., "Carbon nanotube films for damping applications," Adv Mater 14(2002) pp. 997-1000. 
Force field, PVT diagram and cyclization behaviour,” Polymer International 44 (1997) pp. $311-330$

[19] Iijima, S., and Ichihashi, T., 1993, "Single-Shell Carbon Nanotubes of 1-nm Diameter," Nature (London), 363, pp. 603-605.

[20] Bethune, D. S., Kiang, C. H., de Vries, M. S., Gorman, G., Savoy, R., Vazquez, J., and Beyers, R., 1993, "Cobalt-Catalyzed Growth of Carbon Nanotubes With SingleAtomic-Layer Walls," Nature London, 363, pp. 605-607.

[21] Dresselhaus, M. S., Dressehaus, G., and Saito, R., 1995, "Physics of Carbon Nanotubes," Carbon, 33(7), pp. 883-891.

[22] Ebbesen, T. W., 1996, “Carbon Nanotubes," Phys. Today, 49(6), pp. 26-32.

[23] Yakobson, B. I., and Richard, E. S., 1997, "Fullerene Nanotubes: C1,000,000 and Beyond," Am. Sci., 85, pp. 324-337.

[24] Ball, P., 2001, "Roll Up for the Revolution," Nature (London), 414, pp. 142-144.

[25] Thostenson, E. T., Ren, Z. F., and Chou, T. W., 2001, "Advances in the Science and Technology of Carbon Nanotubes and Their Composites: A Review," Compos. Sci. Technol., 61, pp. 1899-1912.

[26] Baughman, R. H., Zakhidov, A. A., and de Heer, W. A., 2002, "Carbon Nanotube-Route Toward Applications,” Science, 297, pp. 787-792.

[27] Lau, K. T., Gu, C., and Hui, D., 2006, "A Critical Review on Nanotube and Nanotube/Nanoclay Related Polymer Composite Materials," Composites, Part B, 37, pp. $425-436$.

[28] Wang, C. M., Zhang, Y. Y., Xiang, Z., Reddy, J. N., "Recent Studies on 
Buckling of Carbon Nanotubes", APPLIED MECHANICS REVIEWS V63 Issue:

3 Article Number: 030804

[29] Qian, D., Wagner, G. J., Liu, W. K., Yu, M. F., and Ruoff, R. S., 2002, “Mechanics of Carbon Nanotubes," Appl. Mech. Rev., 55, pp. 495-533.

[30] Saito, Y., Yoshikawa, T., Bandow, S., Tomita, M., and Hayashi, T., 1993

“Interlayer Spacing in Carbon Nanotubes," Phys. Rev. B, 48, pp. 1907-1909.

[31] Charlier, C., and Michenaud, J. P., 1993, "Energetics of Multilayered Carbon Tubules," Phys. Rev. Lett., 70, pp. 1858-1861.

[32] Kiang, C. H., Endo, M., Ajayan, P. M., Dresselhaus, G., and Dresselhaus, M. S., 1998, “Size Effects in Carbon Nanotubes,” Phys. Rev. Lett., 81, pp. 1869-1872.

[33] Coleman, J N., Khan, U., Blau, W J., Gun'ko, Y K., "Small but strong: A review of the mechanical properties of carbon nanotube-polymer composites," Carbon, 2006; v 44; p 1624-1652

[34] Lu JP. "Elastic properties of single and multilayered nanotubes". J Phys Chem Solids 1997;58(11):1649-52.

[35] Treacy MMJ, Ebbesen TW, Gibson JM. “Exceptionally high Young's modulus observed for individual carbon nanotubes". Nature 1996; 381(6584):678-80.

[36] Wong EW, Sheehan PE, Lieber CM. "Nanobeam mechanics: elasticity, strength, and toughness of nanorods and nanotubes". Science 1997;277:1971-5.

[37] Jin Z, Pramoda K, Xu G, Goh SH. "Dynamic mechanical behavior of meltprocessed multi-walled carbon nanotube/poly(methyl methacrylate) composites". Chem Phys Lett 2001;337(1-3):43-7.

[38] Andrews R, Jacques D, Qian DL, Rantell T. "Multiwall carbon nanotubes: 
synthesis and application". Acc Chem Res 2002;35(12): 1008-17.

[39] Thostenson ET, Chou T-W. "Aligned multi-walled carbon nanotube-reinforced composites: processing and mechanical characterization". J Phys D: Appl Phys 2002(16):L77-80.

[40] Lourie, O., Cox, D. M., and Wagner, H. D., 1998, "Buckling and Collapse of Embedded Carbon Nanotubes,” Phys. Rev. Lett., 81, pp. 1638-1641.

[41] Bower, C., Rosen, R., Jin, L., Han, J., and Zhou, O., 1999, "Deformation of Carbon Nanotubes in Nanotube-Polymer Composites,” Appl. Phys. Lett., 74, pp. $3317-3319$

[42] Waters, J. F., Riester, L., Jouzi, M., Guduru, P. R., and Xu, J. M., 2004, "Buckling Instabilities in Multiwalled Carbon Nanotubes Under Uniaxial Compression,” Appl. Phys. Lett., 85, pp. 1787-1789.

[43] Waters, J. F., Guduru, P. R., Jouzi, M., Xu, J. M., Hanlon, T., and Suresh, S., 2005, "Shell Buckling of Individual Multi-Walled Carbon Nanotubes Using Nanoindentation,” Appl. Phys. Lett., 87, p. 103109.

[44] Thostenson ET, Chou TW., "Nanotube buckling in aligned multi-wall carbon nanotube composites," Carbon 2004: 42: 3015-3018.

[45] Ecklund PC, Holden JM, Jishi RA. "Vibrational modes of carbon nanotubes; spectroscopy and theory". Carbon 1995;33(7):959-72.

[46] Jorio A, Pimenta MA, Souza Filho AG, Saito R, Dresselhaus G, Dresselhaus MS. "Characterizing carbon nanotube samples with resonance Raman scattering". New J Phys 2003;5:139.1-139.17. 
[47] Dresselhaus MS, Jorio A, Souza Filho AG, Dresselhaus G, Saito R. "Raman spectroscopy of one isolated carbon nanotube". Physica B 2002;323:15-20.

[48] Dresselhaus MS, Dresselhaus G, Jorio A, Souza AG, Samsonidze GG, Saito R. "Science and applications of single nanotube Raman spectroscopy". J Nanosci Nanotechnol 2003;3(1-2):19-37.

[49] "Materials Studio: Materials Science Modeling and Simulations," http://accelrys.com/

[50] Sun, H., "COMPASS: An ab Initio Force-Field Optimized for Condensed-Phase Applications - Overview with Details on Alkane and Benzene Compounds," J. Phys. Chem. B, 1998, 102 (38), pp 7338-7364

[51] “Compass," http://accelrys.com/

[52] IUPAC, "Van der Waals forces," Compendium of Chemical Terminology, 2nd ed. (the "Gold Book") (1997). Online corrected version: (1994)

[53] Dzyaloshinskii, I E; Lifshitz, E M; Pitaevskii, Lev P (1961). "General Theory of van der Waals Forces". Soviet Physics Uspekhi 4: 153.

[54] Lennard-Jones, J. E. (1924), "On the Determination of Molecular Fields", Proc. R. Soc. Lond. A 106 (738): 463-477

[55] Qian D, Wagner JG, Liu WK, Yu MF, Ruoff RS. "Mechanics of carbon nanotubes”. Appl Mech Rev 2002;55(6):495-533.

[56] Dikin DA, Chen X, Ding W, Wagner G, Ruoff RS. "Resonance vibration of amorphous $\mathrm{SiO} 2$ nanowires driven by mechanical or electrical field excitation". J Appl Phys 2003;93(1):226-30 
[57] Blevins RD. "Formulas for natural frequency and mode shape". New York: Van Nostrand Reinhold; 1979.

[58] Yoon J, Ru CQ, Mioduchowski A. "Timoshenko-beam effects on transverse wave propagation in carbon nanotubes". Composites B2004;35:87-93.

[59] Yoon J, Ru CQ, Mioduchowski A. "Terahertz vibration of short carbon nanotubes modeled as Timoshenko beams". J Appl Mech 2005;72(1):1017.

[60] Yoon J, Ru CQ, Mioduchowski A. "Vibration of an embedded multiwall carbon nanotube”. Compos Sci Technol 2003;63: 1533-42.

[61] Arash B, Ansari R. "Evaluation of nonlocal parameter in the vibrations of singlewalled carbon nanotubes with initial strain”. PHYSICA E-LOW-DIMENSIONAL SYSTEMS \& NANOSTRUCTURES 2010: 42: 2058-2064

[62] Dereli G, Ozdogan C. "Structural stability and energetics of singlewalled carbon nanotubes under uniaxial strain". Phys Rev B 2003;67(3):035416.

[63] Sanchez-Portal D, Artacho E, JSoler JM. "Ab initio structural, elastic and vibrational properties of carbon nanotubes". Phys Rev B 1999;59(19):12678-88 